\title{
Idiographic Personality Coherence: A Quasi Experimental Longitudinal ESM Study
}

\author{
Emorie D Beck \\ Feinberg School of Medicine, Northwestern University \\ Joshua J Jackson \\ Washington University in St. Louis
}

In press at the European Journal of Personality.

Authors Note. Correspondence concerning this article should be addressed to Emorie D Beck, 633 N St. Clair St., Chicago, IL 60611. Email: emorie_beck@ northwestern.edu. The preparation of this manuscript was supported by a grant from the National Science Foundation to Joshua Jackson (BCS-1125553). Data collection was supported by a Society for Personality and Social Psychology Inside the Grant Panel grant awarded to Emorie Beck. Emorie Beck was supported by National Institute on Aging Grants T32 AG00030-3, R01-AG067622, and R01AG018436. 


\begin{abstract}
Personality is a study of persons. However, persons exist within contexts, and personality coherence emerges from persons in contexts. But persons and environments bidirectionally influence each other, with persons selecting into and modifying their contexts, which also have lasting influences on personality. Thus, environmental change should produce changes in personality. Alternatively, environmental changes may produce few changes. This paradoxical viewpoint is based on the idea that novel environments have no predefined appropriate way to behave, which allows preexisting personality systems to stay coherent (Caspi \& Moffitt, 1993). We test these two perspectives by examining longitudinal consistency idiographic personality coherence using a quasi-experimental design $(\mathrm{N}=50$; total assessments $=5,093)$. Personality coherence was assessed up to one year before the COVID-19 pandemic and again during lockdown. We also test antecedents and consequences of consistency, examining both what prospectively predicts consistency as well as what consistency prospectively predicts. Overall, consistency was modest but there were strong individual differences, indicating some people were quite consistent despite environmental upheaval. Moreover, there were relatively few antecedents and consequences of consistency, with the exception of some goals and domains of satisfaction predicting consistency, leaving open the question of why changes in coherence occurs.
\end{abstract}

Keywords: idiographic, personality, consistency, coherence, COVID-19 


\section{Idiographic Personality Coherence: A Quasi Experimental Longitudinal ESM Study}

Since its beginnings, the field of personality has fundamentally been a science of persons, attempting to understand patterns in what individuals do, why they do them, and their stability over time (e.g., Allport, 1921, 1937; Murray, 1938) - that is, personality coherence. But environments have also been fundamental in understanding personality, even in the earliest definitions of personality. Despite this, the bulk of personality scholarship pits persons and environments against one another other with no clear victor (e.g., Sherman et al., 2015), which may be a fool's errand all-together (e.g., Mischel \& Shoda, 1995). The continuing struggle is fueled by the difficulty of disentangling persons from their environments, which appear to share bi-directional relationships (e.g., Rauthmann et al., 2017). Indeed, there is evidence that people select into (e.g., Emmons \& Diener, 1986) and modify their environments (e.g., Roberts, Donnellan, \& Hill, 2012) as well as evidence that environments have long-lasting influences on cognition, emotion, and behavior (e.g., Briley \& Tucker-Drob, 2017).

The tension between persons and environment came to a head in the latter half of the 20th century in the so-called Person-Situation Debate (see Beck \& Jackson, 2020a, for a summary). At the time, there was disagreement about the kinds of coherence personality should exhibit and the degree of short-term and long-term consistency it should exhibit over time. On the one hand, nomothetic, trait approaches to personality emphasized that personality coherence is exhibited in tendencies to exhibit similar types and degrees of trait-relevant behaviors across situations over long time periods (e.g., Bem \& Funder, 1978; Epstein, 1979). On the other, some social cognitive approaches to personality emphasized that coherence is exhibited in the stability of personality within specific situations - that is, in the if...then relationships that characterized an individual across behaviors and contexts (e.g., Mischel, 1973; Mischel \& Peake, 1982; Wright \& 
Mischel, 1987). Since the debate began, there have been declarations of its end (e.g., Fleeson \& Noftle, 2008) as well as retrospectives of it (Donnellan, Lucas, \& Fleeson, 2009). However, at least one central question remains unaddressed: if a person's situation changes, does their personality coherence - operationalized either as traits or as collections of if...then relationships - change or does it remain consistent over the long-term? Although a few previous studies investigated personality across two different situations or events, these situations are confounded with personality through situation selection processes (Furr \& Funder, 2004). As a result, there has been limited evidence suggesting that personality operates as a coherent system in the face of environmental change. The current study uses the novel SARS-CoV-2 virus that results in the COVID-19 disease as an exogenous manipulation to examine how changing situations influences consistency and change in idiographic personality coherence.

\section{Persons and Environments}

Although the divergent perspectives of the Person-Situation Debate disagreed on how to conceptualize personality, they agreed that environments are catalysts of both personality coherence and change. As such, both theoretical perspectives would make the claim that changes in environments lead to changes in personality coherence.

Nomothetic perspectives to personality conceptualize personality as traits, or dispositional patterns of thoughts, feelings, and behaviors (Roberts \& Jackson, 2008), in which levels on a trait dimension differentiate individuals. Coherence, in this regard, is demonstrated through population-level patterns of longitudinal consistency (i.e. minimal changes). Most commonly, such longitudinal consistency is examined using variable-centered rank-order consistency/change and less commonly through person-centered ipsative consistency/change (typically examined as correlations of one individual's profile of trait scores across two measurement occasions). 
Indeed, traits show great rank-order consistency (e.g., Damien, Spengler, Sutu, \& Roberts, 2019) and ipsative consistency (Robins, Fraley, Roberts, \& Trzesniewski, 2001) across years and even decades. Because rank-order and ipsative consistency suggest that traits exist across time and environments, these findings are often offered as evidence of the utility of personality traits as constructs and of personality's coherence. In other words, because (1) traits reliably differentiate individuals and (2) individuals show relatively stable levels of personality traits both at single measurement occasions as well as across them, traits capture coherence in personality in their ability to rank-order individuals, regardless of the environments in which they are situated.

Both rank-order and ipsative estimates of trait consistency/change de-emphasize the importance of the environment, instead focusing on coherence as the consistency (and lack of large-scale change) of traits despite changing environments across time. Notwithstanding, environments are the favored explanation of personality change - through the social roles people take (Lodi Smith \& Roberts, 2007) and the events they experience (e.g., Bleidorn, Hopwood, \& Lucas, 2018) - with behavior genetic models of personality development finding that environments almost entirely mediate changes in personality traits (Bleidorn, Kandler, Riemann, Angleitner, \& Spinath, 2012).

Many social-cognitive perspectives, in contrast to trait perspectives, conceptualize personality as persons-in-environments, which contextualizes how an individual behaves within and across contexts, with an emphasis on that individual (Beck \& Jackson, 2020ab; Cervone, 2005; Cervone \& Shoda, 1999; Danvers, Wundrack, \& Mehl, 2020; Fournier, Moscowitz, \& Zuroff, 2008 Wright \& Mischel, 1987). From this perspective, personality is defined in terms of if...then contingencies, or the conditional probability that an individual engages in a behavior in within a context $(p(\mathrm{~B} \mid \mathrm{C})$; Wright $\&$ Mischel, 1987). Rather than viewing personality as traits that 
represent collections of behaviors, assuming that personality coherence is found in the ability of those aggregated behaviors to rank order individual over time, these social-cognitive approaches treat personality - and personality coherence - as collection of if...then contingencies, which, in their simplest form, can be represented as a profile of contingencies. In other words, coherence exists within the stability of if...then patterns an individual exhibits, regardless of (1) whether such a pattern is shared by others and (2) whether seemingly incoherent behaviors, like being "shy" but also "skilled at public relations" are reported as important to a person (Cervone \& Shoda, 1999). Consistency and change, in turn, are viewed as the longitudinal consistency or change of if...then contingencies (i.e. coherence) across different contexts over time, rather than as an average tendency collapsed across situations as in trait perspectives.

Developmentally, if...then contingencies are learned over time. When new contexts are introduced, an individual must cognitively appraise the new context and generate a behavioral response from their existing set of behaviors, or so-called behavioral competencies (Mischel, 1973; Mischel \& Shoda, 1995). However, the environment is still playing an important role, and changes to the environment should result in changes in personality expression. In the simplest sense, introducing a new situation means there is no learned contingency to guide behavioral responses. In other words, there is no coherent personality manifestation an individual can exhibit. Thus, there is only the situation to guide behavior, and new situations should induce changes in personality coherence.

Thus, trait and social-cognitive perspectives both posit that changing environments should result in changing patterns of coherence - that is, in reduced personality consistency (i.e. in more change). Despite this, there have been relatively few tests of personality coherence and consistency across major environmental changes, partially due to the difficulty of capturing 
environmental changes. Indeed, there is heterogeneity in how, when, and why environmental changes occur, and individuals rarely encounter the same events at the same time (see Luhmann, Orth, Specht, Kandler, \& Lucas, 2014). Thus, most research examining how environments influence personality must assume that events experienced by different individuals at different times and locations and with different precursors are similar.

\section{An Idiographic Approach to Personality Coherence}

One reason for the continuing tensions between nomothetic trait approaches and social cognitive approaches lies in the disparate goals of the two approaches, which mirror the varied goals of personality psychology more broadly (Mõttus et al., 2020). On the one hand, personality psychology seeks to describe broad patterns in how individuals differ from one another on a number of shared characteristics and what such characteristics predict (often examined through a variable-centered approach). On the other, personality psychology seeks to understand an individual in their own terms and using their own lived experiences (a person-centered approach). Here, the focus is not describing and predicting broad patterns in a population but in describing and explaining how and why an individual engages in unique patterns of experiences and behaviors. While nomothetic trait approaches pursue the former, social cognitive approaches pursue the latter.

In studying personality coherence, the social cognitive approaches we draw on (e.g., Mischel \& Shoda, 1995; Wright \& Mischel, 1987) greatly benefit from drawing on idiographic, person-centered approaches. In the simplest case, this is because it allows coherence to focus on contexts, experiences, and behaviors that are critical to an individual and their life. Rather than relying on trait methods' assumptions that coherence is rooted in average tendencies, idiographic methods rely on the life of an individual to dictate what is important. Or, as Gordon Allport said, 
"the method [of dimensional approaches] required it, but the life did not" (Allport, 1968, p. 62). Despite this, idiographic methods have remained difficult to utilize, as the methods necessary to capture the lived experience of an individual are intensive to measure and to model.

Recently, new measurement and modeling techniques have paved new avenues for modeling personality idiographically and using such approaches to understand personality coherence through a new lens. First, the introduction of the Experience Sampling Method (ESM) allows for intensive measurement of an individual by pinging them using a smartphone or other device to respond to a short survey about their momentary experiences. In so doing, an individual is measured in the context of their life, allowing researchers to examine the patterns of if...then contingencies that are central to understanding personality coherence from a social cognitive perspective. Second, new modeling techniques allow researchers to probabilistically model associations both among physical and experiential contexts, behavior, and experiences in everyday life (e.g., Lane, Gates, Pike, Beltz, \& Wright, 2019), a feat whose importance has been touted for decades (e.g., Wright \& Mischel, 1987).

Together, intensive longitudinal data and new modeling techniques allow researchers to understand personality - and personality coherence - as a system of social, psychological, and behavioral processes interacting with one another, with coherence evident in stable patterns among each of these. For example, one recent investigation used longitudinal Experience Samplign Method data collected separately across two years to estimate idiographic personality coherence as a set of relationships among personality manifestations (Beck \& Jackson, 2020b). The recovered set of relationships for each person capture the coherence of their personality that is, the consistent meaningful ways in which they reported their experiences and behaviors across different contexts. In this study, across people, individuals demonstrated substantively 
different patterns of personality coherence, but within-person, their coherence was quite consistent over the two-year period of the study. Idiographic methods such as these can thus serve as a useful and reliable estimator of factors that influence idiographic personality coherence.

\section{A Paradox of Coherence}

Although trait and social cognitive perspectives emphasize that changes in personality coherence are primarily due to changes in the environments individuals inhabit, an alternative perspective argues that new environments could lead to higher levels of consistency. This alternative perspective suggests that behavioral coherence and longitudinal consistency is likely to be seen when there is environmental instability of a particular kind (Caspi \& Moffit, 1993). People rely on their stable aspects of personality when they enter new environments that are novel, do not have well established behavioral norms, or are marked by large amounts of uncertainty. That is, people are likely to default to behaviors that are automatic and most central during times of disruption, falling back on their dispositions for guidance. Consequently, such environmental shifts may exaggerate individual differences between people, increasing coherence and consistency in patterns of behavior rather than attenuating them.

Some evidence exists for this "paradoxical theory" where weak situation social upheaval could lead to greater (or sustained) consistency and largely unchanged patterns of coherence (Caspi \& Moffitt, 2001; Neyer et al., 2014). However, most experiences do not fall under the weak situation umbrella, as experiences that are thought to be of sufficient magnitude to change personality often come with proscribed rules and expectations to guide behavior (e.g., military experience; Jackson et al., 2012). Similarly, most experiences or situations are driven in part by a person's personality, making it difficult to disentangle effects due to the person or situation 
(Funder, 2006). Perhaps the closest test of this longstanding theory has been with the experience of trauma, finding that trauma did not engender decreases in personality consistency (Ogle, Rubin, Siegler, 2014). Despite this, there are, overall, few tests of the consistency of personality coherence in the wake of situation change, leaving whether environmental change produces more or less consistency an open question.

\section{The Present Study}

The COVID-19 outbreak and resulting worldwide lockdowns and social distancing measures provide a unique opportunity to test whether and for whom a broad reaching environmental change is associated with consistency in personality coherence across time. Despite decades of research on how environments influence personality, almost no study to date has tested whether personality remains consistent in the context of major environmental shifts. Indeed, only rarely do populations experience similar events that lead to great contextual changes. The recent COVID-19 pandemic, which has been accompanied by nationwide shutdowns and social distancing measures, thus offers a unique opportunity for studying personality consistency and change. We adopt an idiographic approach to investigate coherence in personality and its consistency across a major disruption. Idiographic perspectives overcome issues of examining nomothetic personality alone and allows situations and dynamic features of personality to be incorporated directly within the model (Beck \& Jackson, 2020abc). Thus, the present study fills a gap in the literature by examining personality coherence changes throughout widespread environmental change during a global pandemic.

Using pre- and post-COVID-19 onset personality data from a longitudinal study that began in 2018, we address three broad questions. First, how much idiographic longitudinal consistency in personality coherence is demonstrated in the wake of a global health crisis? We 
expect that personality coherence will remain quite consistent over this period but that there will be individual differences in consistency. Second, we then examine these individual differences in more detail, asking what are the social, psychological, and academic well-being antecedents of consistent coherence. Finally, we also examine the social, psychological, and academic wellbeing consequences of consistency in the wake on COVID-19.

\section{Method}

This study was preregistered on the Open Science Framework (OSF; https://osf.io/qjs5v) and all data, analysis scripts, and results are available on both the OSF (https://osf.io/qwtu3/) and GitHub (https://github.com/emoriebeck/covid_consistency). All data are completely deidentified. This study was approved by the Institutional Review Board at Washington University in St. Louis (\#201806124), and all data were collected in alignment with the APA ethics code.

\section{Participants}

Participants were 50 (68.5\% female; $\left.\mathrm{M}_{\mathrm{age}}=19.60, \mathrm{SD}_{\mathrm{age}}=1.13\right)$ undergraduates at Washington University in St. Louis who had previously enrolled in a longitudinal personality intervention study. 18 identified as white, 18 as Asian, four as Black, and 11 other race/ethnicity or mixed race/ethnicity (two declined to answer). See Table S1 in the online materials for additional information. Participants who had enrolled in the study between March and December 2019 who had not taken part in the intervention portion of the study were contacted in midMarch 2020 via email to take part in a separate study regarding their experiences in the wake of the university closure and social distancing measures related to COVID-19. Participants completed three waves of trait assessments and 2 waves of experience sampling method (ESM) assessments. More information on the timeline of recruitment and relevant events can be seen in Figure 1. Because the larger study uses rolling recruitment and intentionally oversamples the 
baseline assessments for construct validation purposes, the first wave of both trait and ESM assessments took place between March and December $2019(N=50$, total ESM assessments $=$ 2,911; $M=64.7, S D=22.9, \min =20, \max =109)$. Participants were recontacted on March 16 after the university announced an extended spring break (March 11) but before the university was fully shut down and classes moved online (March 20). The second and third waves of trait assessments took place on March 20-22, 2020, and April 8-10, 2020, respectively, while the second wave of ESM assessments took place from March 23 to April 5, $2020(N=50$, total ESM assessments $=2,182 ; M=48.5, S D=11.3, \min =15, \max =63)$. Participants were compensated for each survey completed, including \$10 each for the first and third waves of trait assessments, $\$ 5$ for the second wave of trait assessments, and \$.50 / ESM assessment (for a maximum of \$30 if completing at least 50 surveys at each wave).

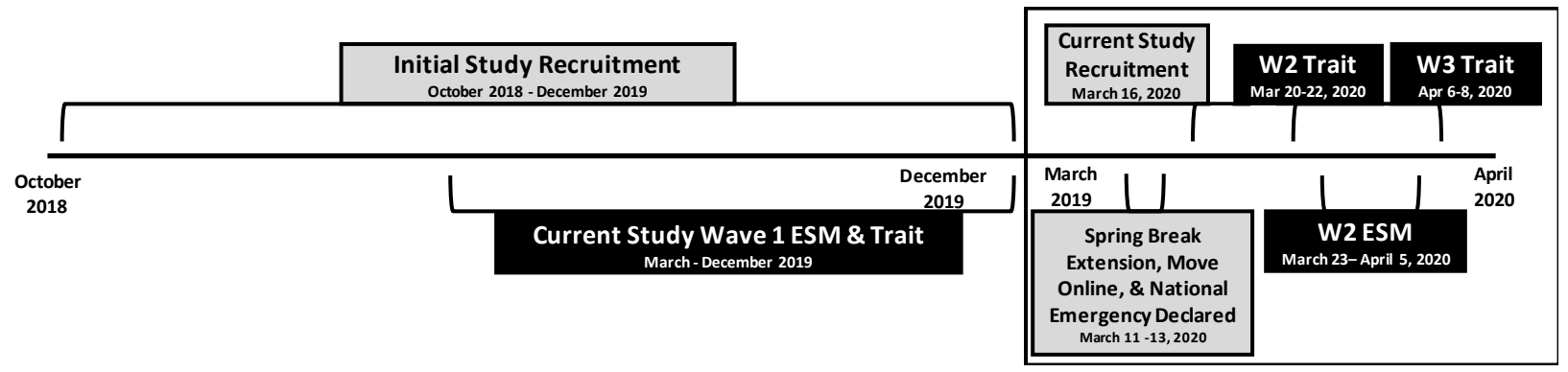

Figure 1. Timeline of data collection, the larger study, University closing, and other COVID-19 relevant events. The right side of the timeline, enclosed in a box, is not to scale in order to outline the full sequence of events.

\section{Measures}

Participants responded to a large battery of trait and ESM measures as part of the larger study. The present study focuses on a smaller subset of measures whose use we preregistered. A full list of the collected measures for the study can be found in supplementary codebooks in the online materials on the OSF and GitHub. The measures collected at each wave were identical, with the exception of the addition of a number of items specific to COVID-19 experiences and 
responses that were included in the third wave of trait measures that were used in the present study only for descriptive purposes. ESM measures were used to estimate idiographic personality and idiographic personality consistency. Trait measures were used as antecedents and consequences of consistency, COVID-19 experiences were used as consequences of consistency. Antecedents and Consequences

Depression. Trait depression was measured using the Center for Epidemiological Studies-Depression scale (CES-D; Radloff, 1977), which is a 20-item scale indexing the number of days in the last week that participants have experienced several symptoms of depression (e.g., loss of sleep, loneliness). Participants responded to each item on a 4-point Likert-like scale from 0 "Rarely or none of the time" to 3 "Most or all of the time."

Loneliness. Loneliness was measured using the 6-item short-form of the UCLA Loneliness Scale (Neto, 1992). This inventory was assessed twice, once as a global measure and once with specific reference to participants' experiences in university. Items assessed different features of loneliness (e.g., "I feel isolated"; "I lack companionship"). Items were responded to on a 4-point Likert-like scale from 0 "I never feel this way" to 3 "I often feel this way."

Satisfaction with Life. Satisfaction with life (SWL) was measured using the 5-item Satisfaction with Life scale (Diener, Emmons, Larsen, \& Griffin, 1985) on a scale from 1 "strongly disagree" to 7 "strongly agree."

Domain Satisfaction. In addition, we assessed satisfaction in nine specific domains, including family, friendships, romantic relationships, community, academics, finances, physical health, psychological health, and hobbies. Each item was responded to on the same 7-point Likert-like scale as the SWL scale from 1 "strongly disagree" to 7 "strongly agree." 
Diligence. Diligence was measured using 11 items from the 48-item Diligence Inventory that were selected by the authors in early 2018 as the most relevant to college undergraduates. Each item is meant to capture student's cognitions (e.g., "I set high standards for myself."), emotions (e.g., I do not get upset over the amount of academic work I have to do.”), and behaviors (e.g., "I do my assignments as soon as I get them.”) surrounding academic work. Each item was responded to on a 5-point Likert-like scale from 1 "not like me at all" to 5 "very much like me."

Goals. Goals were measured using 12 items from a larger set of 26 major life goals used in prior studies (e.g., Roberts \& Robins, 2000). Each item was assessed on a 5-point Likert-like scale from 1 "extremely unimportant" to 5 "extremely important."

Purpose in Life. We assessed purpose in life using the 7-item Purpose in Life subscale of Ryff's (1989) Psychological Well-Being scale (e.g., "I have a sense of direction and purpose in life."). Each item was responded to on a 6-point Likert-like scale from 1 "strongly disagree" to 6 "strongly agree."

COVID-19. We assessed a number of behaviors and experiences relevant to COVID-19, including pre-existing conditions (e.g., diabetes), symptoms of COVID-19 (e.g., shortness of breath), testing and treatment of COVID-19, worry about COVID-19, employment and financial situations relative to COVID-19 (e.g., "Were you previously employed but laid off due to COVID-19?”), and pre- and post-COVID-19 social contact (e.g., "Before/After COVID-19 social distancing measures were put into place, how often did you... wear a mask”). Full detail on the scale and text of each question can be found in the codebook in the online materials. 
Personality. Personality was assessed using the full BFI-2 (Soto \& John, 2017). The scale was administered using a planned missing data design (Revelle et al., 2016). We have previously demonstrated both the between- and within-person construct validity of assessing personality using planned missing designs using the BFI-2 (https://osf.io/pj9sy/). The planned missingness was done within each Big Five trait separately, with three items from each trait included at each timepoint (75\% missingness). Each item was answered relative to what a participant was just doing on a 5-point Likert-like scale from 1 "disagree strongly" to 5 "agree strongly." Items for each person at each assessment were determined by pulling 3 numbers (1 to 12) from a uniform distribution. The order of the resulting 15 items were then randomized before being displayed to participants.

\section{Procedure}

Participants in this study were drawn from a larger personality study. All responded to two types of surveys: trait and state (Experience Sampling Method; ESM) measures, for which they were paid separately. Participants completed three waves of trait measures and two waves of state measures. For the first two waves, trait surveys were collected immediately before beginning the ESM protocol.

\section{Wave 1}

For the first wave, participants were recruited from the psychology subject pool at Washington University in St. Louis. Participants were told that the study posted on the recruitment website was the first wave of a longer longitudinal study they would be offered the opportunity to take part in.

Participants were brought into the lab between October 2018 and December 2019, where a research assistant or the first author explained the study procedure to them and walked them 
through the consent procedure. If they consented, participants were led to a room where they could fill out a form to opt into the ESM portion of the study. They then completed baseline trait measures using the Qualtrics Survey Platform. After, the participants were debriefed, paid \$10 in cash and, if they opted into the ESM portion of the study, the ESM survey procedure was explained to them.

Participants then received ESM surveys four times per day for two weeks ( $\operatorname{target} n=56$ ). The survey platform was built by the first author using the jsPsych library (De Leeuw, 2015). Additional JavaScript controllers were written for the purpose of this study and are available on the first author's GitHub. Start times were based on times that participants indicated they would like to receive their first survey based on their personal wake times. Surveys were sent every 4 hours, meaning that the surveys spanned a 12-hour period from the start time participants indicated. Participants received their first survey at their chosen time on the Monday following their in-lab session. They were compensated $\$ .50$ for each survey completed for a maximum of \$28. To incentivize responding, participants who completed at least 50 surveys received a "bonus" for a total compensation of \$30, which was distributed as an Amazon Gift Card. ${ }^{1}$

\section{Waves 2 and 3}

Additional waves of data were completed remotely online. Participants who completed baseline trait and ESM assessments between March and December 2019 were contacted via email by the first author on Monday, March 16, 2020. On Friday, March 20, participants who responded then received an email with a link to a Qualtrics survey on with which they completed a second set of trait measures for which they were compensated $\$ 5$ (wave 2). The following

\footnotetext{
${ }^{1}$ In Wave 1 ESM assessments, some participants completed additional assessments for the purposes of validating the planned missing procedure across different within-person sample sizes. In Wave 2, because we did not require participants to answer within a specific window or close surveys after a response, a few participants surpassed the maximum of 56 assessments by unprompted completion of additional surveys during or after the survey period.
} 
Monday, March 23, after being given the opportunity to modify their start time to accommodate different time zones or conditions, these participants then received a second round of ESM surveys. As before, participants received these 4 times per day, 4 hours apart, for 2 weeks (March 23 to April 5) and were compensated $\$ .50$ per survey for up 50 surveys, and $\$ 30$ total for responding to more than that. At the end of the 2 weeks, on April 6, participants received an email link containing a new Qualtrics link to the next round of trait measures (wave 3), which also included additional measures related to COVID-19 experiences. For completing this survey, participants were compensated \$1. Each of these later surveys were paid as a single Amazon Gift Card sent to participants at an email of their choice.

\section{Analytic Plan}

\section{Estimating Idiographic Models}

First, we prepared the data according to a series of preregistered steps. Trait measures were composited for each wave separately. ESM data were cleaned in several steps. First, based on previous research (e.g., Beck \& Jackson, 2020b) and to avoid having more predictors than observations, we excluded participants with less than 20 responses. Second, we composited personality data within the $15 \mathrm{BFI}-2$ facets for each person in each wave at each measured time point. Because previous work indicated that these imputed data show good between-person structure and strong convergence with both raw data and trait data (https://osf.io/pj9sy/), these data were then multiply imputed using the amelia (Honaker, King, \& Blackwell, 2011) package in $\mathrm{R}$ (R Core Team, 2019). Because almost all participants in ESM studies inevitably had missed assessments and overnight periods, we then padded the missing assessments and overnight periods with empty rows. ${ }^{2}$

\footnotetext{
${ }^{2}$ In the preregistration, we planned to use cubic spline interpolation to normalize responses. However, this method resulted in hugely improbable values, so we did not normalize responses but simply controlled for overnight periods
} 
Next, we estimated idiographic personality structure using the GIMME procedure, which is a data-driven procedure for estimating both group-level and idiographic relationships in time series data (Lane, Gates, Pike, Beltz, \& Wright, 2019). As currently implemented in the gimme package (Lane, Gates, Molenaar, Hallquist, \& Pike, 2016) in R, the procedure estimates a series of unified structural equation model (uSEM) for each person and constructs a group-level structure based on the individual-level models. uSEM uses an iterative procedure for retaining pathways in the model using Lagrange multiplier tests. The GIMME procedure begins by estimating the pathways to be retained at the group-level (i.e. in all individual-level models) by estimating individual-level models and retaining group-level pathways for those paths were shared by $75 \%$ of participants. Starting with a null model, pathways are iteratively added to the group-level structure (i.e. in all participants' final unique models) when the largest proportion of individuals (above a chosen threshold, $75 \%$ by default) show a better model fit according to the Lagrange multiplier tests. This procedure is continued until no additional pathways improve fit above the threshold. Idiographic models are then built using a similar procedure, with the exception that each step of the Lagrange multiplier test is only based on the target individual (rather than the set of individual Lagrange multiplier tests) and begins with all paths retained at the group-level structure as the null model. The iterative procedure continues for each person until the procedure indicates that no pathways improve model fit. For this procedure, each of the 15 personality imputed indicators were included. This procedure was repeated for each wave of ESM data. ${ }^{3}$

in the main reported analyses. Doing so means that lagged values are not regressed across days or "missing" overnight periods.

${ }^{3}$ As a robustness test, we also tested models that included an exogenous cumulative time variable. This means that the cumulative time is used to predict all variables but is not predicted by them in order to detrend the data. In addition, we tested a simple linear interpolation method for dealing with unequal intervals. That is, after padding, we 
The GIMME procedure resulted in an asymmetric, rectangular matrix of coefficients from each participant's uSEM model, where rows are outcome variables and columns are predictor variables. The number of rows is equal to the number of indicators, which, in the present paper, is 15 , while the number of columns is equal to $2 \mathrm{x}$ the number of indicators (30) because lagged predictors are also included in the model. Based on prior work (Beck \& Jackson, 2020b), we investigated the results as contemporaneous (within-time), lagged (across-time; all associations between current and previous time point indicators). In the simplest case, contemporaneous associations test whether observed levels of one indicator at time $t$ track with the levels of another indicator measured at the same time point $t$, while lagged associations test whether levels of one indicator at time $t$ track with previous time point $(t-1)$ levels of the same or another indicator.

\section{Personality Consistency}

Once the GIMME models are estimated, we estimated idiographic personality consistency by estimating the profile correlation of all possible pathways for each person contemporaneously, lagged. Profile correlations are a measure of longitudinal ipsative consistency. High positive ipsative consistency suggests the profiles are very similar (i.e. parallel) over time. Zero correlations suggest that there is no pattern in how the two profiles differ. Negative correlations mean there is a consistent pattern of reversals (i.e. high estimates at one timepoint are now low and vice versa) and are generally less common. They do not speak to

controlled for unequal intervals between observed measurement occasions by adjusting consecutive estimates such that all lagged model estimates were based on 4 hour intervals:

$x_{t}^{\prime}=\frac{x_{t}-x_{t-1}}{t_{t}-t_{t-1}} * 4+x_{t-1}$, where $x_{t}$ indicates observation $t$ of the observed variable $\mathrm{x}, x_{t-1}$ is the prior measurement occasion observation of variable $x$ (including missing observations), $t_{t}$ and $t_{t-1}$ are time stamps of the current and previous measurement occasions (or expected observations or overnight periods). When $t_{t}-t_{t-1}<4$, this results in an inflated value $x_{t}^{\prime}$, while when $t_{t}-t_{t-1}>4$, this results in a reduced value $x_{t}^{\prime}$.

Parallel results to all those presented in the main results of the present study are included in the online materials. 
specific levels, but patterns of levels over time. To do this, for each participant separately, we took the asymmetric rectangular matrix estimated for each person from the GIMME procedure at wave 1 and correlated it with their corresponding GIMME matrix at wave 2. Correlations were estimated using the cor. ci ( ) function in the psych package (in order to estimate bootstrapped confidence intervals of the correlations; Revelle, 2017). We tested these both for contemporaneous and lagged associations separately because previous research suggests that lagged associations are less consistent than contemporaneous ones (Beck \& Jackson, 2020b).

In addition, because we used a larger number of indicators than the previous study $(15 \mathrm{v}$ 9) and a different core modeling technique (GIMME v graphicalVAR; Wild et al., 2010), we additionally repeated this procedure for a subset of 9 items matched to those from Beck and Jackson (2020b), who used data from a different study on a similar population. This allows us to compare whether the consistency observed in the present study in the wake of COVID-19 is similar to that observed outside of the environmental presses created by a global event.

\section{Antecedents of Consistency}

Next, we addressed Aim 2, testing for antecedents of consistency (i.e. what prospectively predicts individual differences in idiographic longitudinal consistency of personality). To do so, we used the consistency estimates from Aim 1 as well as baseline estimates of target antecedents from the first two waves of trait estimates, both of which were collected prior to the second wave of experience sampling data. In addition, we used items in which we asked participants about their pre- and post-COVID relationships, worry, and employment. Consistency was transformed to $z$-scores using Fisher's $r$-to- $z$ transformation to control for the non-normality of correlations.

We preregistered a number of antecedents that we expected to predict consistency. These were a subset of measures from larger study from which these data were drawn, which focused 
on the social and academic experiences of college students, chosen because of the authors' perceived relevance to consistency in personality. Thus, the antecedents largely focused on goals and satisfaction within these domains, as well as other trait-level individual difference characteristics, like loneliness, diligence, purpose in life, and depression. Although preregistered, these analyses were largely exploratory because the dearth of studies of idiographic consistency meant we had no strong predictions about the direction or magnitude of the associations. For each of these, we tested whether wave 1 or 2 baseline measures predict consistency using Bayesian multiple regression using the brms (Bürkner, 2016) package in R. In addition, we tested whether a linear combination of the 2 (i.e. an interaction) between the estimates at each wave out predict the estimates alone. In other words, we tested whether changes between wave 1 and wave 2 predicted consistency. Because participants have different measurement intervals between ESM survey periods, we controlled for the elapsed time between the waves of ESM. We used weakly regularizing priors for all analyses and 3000 samples (1000 warmup samples). Model fit and convergence were tested by looking at $\hat{R}$ values. The critical test for these models was examining the target (1) wave 1 baseline measures (controlling for interval), (2) wave 2 baseline measures (controlling for interval and wave 1), and (3) the interaction between wave 1 and wave 2 baseline measures (i.e. change across waves; controlling for interval, wave 1, and wave 2) using the brms package.

\section{Consequences of Consistency}

In a final step, we addressed Aim 3, testing for consequences of consistency (i.e. what do individual differences in idiographic longitudinal consistency of personality prospectively predict) for the same measures we tested as antecedents in Aim 2, as well as the remainder of the questions we asked participants about their COVID-19 related experiences that had sufficient 
variability to conduct analyses (e.g., note the few to no participants who reported pre-existing conditions and COVID-19 symptoms in Table 1$)^{4}$. These were tested using multiple regression in the brms package in R. We controlled for baseline (wave 1) measures of each target outcome (where available) as well as the interval between ESM waves.

We also include a number descriptives of the sample, including COVID-19 diagnoses, tests, symptoms, and pre-existing conditions.

\section{Results}

\section{Descriptives}

First, we examined the descriptive statistics of the antecedents and consequences as well as COVID-19 related experiences. The descriptives for binary Yes-No questions related to COVID-19-related behavior, treatments, and pre-existing conditions, shown in Table 1, served as the basis of whether they were included in subsequent analyses. As is clear in the table, the participants had few pre-existing conditions and little direct contact with COVID-19 during these early weeks of the shutdown. However, several participants had personally or through family members had their employment or financial situation impacted by COVID-19. Participants also reported engaging in a number of behaviors, like wearing a mask and avoiding gyms, to mitigate their risk of contracting COVID-19. Table 2 presents participants' social contact before and after COVID-19 lockdowns. Participants spent much more time with family in person and more time with friends on video chat following the onset of COVID-19, serving as a validation check for environmental change.

\section{Table 1}

Frequencies of COVID-19-Related Experiences and Pre-Existing Conditions

Item Text

\# No \# Yes \% Yes

\section{COVID-19 Behaviors}

\footnotetext{
${ }^{4}$ Testing of COVID-related data from Wave 3 surveys was not preregistered but added to better capture environmental changes due to COVID-19 based on helpful feedback from a reviewer.
} 
Table 1

Frequencies of COVID-19-Related Experiences and Pre-Existing Conditions

Item Text

\# No \# Yes \% Yes

Going to the other side of the sidewalk?

$113777.08 \%$

Washing hands more than usual?

$6 \quad 4287.50 \%$

Wearing a mask?

$22 \quad 2654.17 \%$

Working out inside or at home rather than a gym or outside?

$9 \quad 3981.25 \%$

\section{COVID-19 Employment}

Are you currently employed?

$37 \quad 1122.92 \%$

Did you or your family have money concerns before 2020 ?

$35 \quad 1327.08 \%$

Do you or your family now have money concerns due to COVID-19? $\quad 30 \quad 18 \quad 37.50 \%$

Has a family member been laid off due to COVID-19?

Were you previously employed but laid off due to COVID-19? $\quad 40 \quad 816.67 \%$

\section{COVID-19 Symptoms}

\begin{tabular}{|c|c|c|}
\hline Dry cough? & 46 & $24.17 \%$ \\
\hline Fever? & 46 & $24.17 \%$ \\
\hline Other (specify) & 47 & $12.08 \%$ \\
\hline Pain or pressure in the chest? & 45 & $36.25 \%$ \\
\hline Shortness of breath? & 48 & $00.00 \%$ \\
\hline \multicolumn{3}{|l|}{ COVID-19 Treatments } \\
\hline Have you been tested for COVID-19? & 47 & $12.08 \%$ \\
\hline Have you been treated for COVID-19? & 48 & $00.00 \%$ \\
\hline Have you contacted a doctor because of COVID-19? & 45 & $36.25 \%$ \\
\hline Have you ever thought you had COVID-19? & 38 & $1020.83 \%$ \\
\hline \multicolumn{3}{|l|}{ Pre-existing Conditions } \\
\hline Any Heart Disease or Chronic Issue & 48 & $00.00 \%$ \\
\hline Any Upper Respiratory Disease or Chronic Issue & 48 & $00.00 \%$ \\
\hline Asthma & 47 & $12.08 \%$ \\
\hline Cancer & 48 & $00.00 \%$ \\
\hline Chronic Bronchitis & 47 & $12.08 \%$ \\
\hline Diabetes & 48 & $00.00 \%$ \\
\hline High Blood Pressure & 48 & $00.00 \%$ \\
\hline Kidney Disease & 48 & $00.00 \%$ \\
\hline Liver Disease or Chronic Issue & 48 & $00.00 \%$ \\
\hline Other: Specify & 47 & $12.08 \%$ \\
\hline
\end{tabular}

Table 2

Descriptives of COVID-19-Social Contact

Item Text

M SD $\quad$ Min $\quad$ Max

Prior to COVID-19, how often did you...

Spend time with family in person?

Spend time with family on the phone?

Spend time with family on video chat?

Spend time with friends in person?
1.73

3.02

2.31

5.25

2.28

1.36

1.70

1.19
$612.50 \%$

$24.17 \%$

$4.17 \%$

$2.08 \%$

$6.25 \%$

$0.00 \%$ 
Table 2

Descriptives of COVID-19-Social Contact

\begin{tabular}{ccccc} 
Item Text & M & SD & Min & Max \\
\hline Spend time with friends on the phone? & 3.23 & 1.67 & 0 & 6 \\
Spend time with friends on video chat? & 2.44 & 1.81 & 0 & 6
\end{tabular}

Following COVID-19, how often did you...

\begin{tabular}{lllll}
\hline Spend time with family in person? & 4.46 & 2.53 & 0 & 6 \\
Spend time with family on the phone? & 2.32 & 1.79 & 0 & 6 \\
Spend time with family on video chat? & 2.06 & 1.84 & 0 & 6 \\
Spend time with friends in person? & 1.60 & 2.25 & 0 & 6 \\
Spend time with friends on the phone? & 3.79 & 1.81 & 0 & 6 \\
\multicolumn{7}{c}{ Relative to before COVID-19, how often do you... } \\
\hline Spend time with family in person? & 4.23 & 1.15 & 1 & 5 \\
Spend time with family on the phone? & 2.89 & 1.26 & 1 & 5 \\
Spend time with family on video chat? & 2.98 & 1.26 & 1 & 5 \\
Spend time with friends in person? & 1.38 & .97 & 1 & 5 \\
Spend time with friends on the phone? & 3.83 & .87 & 1 & 5 \\
Spend time with friends on video chat? & 4.26 & .87 & 1 & 5
\end{tabular}

The scale for pre- and post-COVID-19 social experiences was: $0=$ Never, $1=$ Once a month, $2=$ A few times a month, $3=$ Once a week, $4=2-3$ times week, 5=4-6 times a week, $6=$ every day. The scale for comparing changes was: $1=$ Much less often, $2=$ Slightly less often, $3=$ No change, 4 = Slightly more often, $5=$ Much more often

Table 3

Descriptive Statistics of Trait-Level Characteristics Across Waves

\begin{tabular}{|c|c|c|c|c|c|c|c|c|c|}
\hline \multirow[b]{2}{*}{ Trait } & \multicolumn{3}{|c|}{ Wave 1} & \multicolumn{3}{|c|}{ Wave 2} & \multicolumn{3}{|c|}{ Wave 3} \\
\hline & M (SD) & Range & $\alpha$ & M (SD) & Range & $\alpha$ & M (SD) & Range & $\alpha$ \\
\hline Depression & $1.43(.56)$ & $.60-2.75$ & .92 & $1.56(.56)$ & $.60-2.70$ & .91 & $1.57(.60)$ & $.65-3.05$ & .94 \\
\hline Diligence & $3.07(.75)$ & $1.36-4.73$ & .83 & $3.23(.74)$ & $1.91-5.00$ & .84 & $3.16(.68)$ & $1.82-5.00$ & .79 \\
\hline Career Goals & $4.56(.74)$ & $2.00-5.00$ & & $4.56(.71)$ & $2.00-5.00$ & & $4.65(.67)$ & $2.00-5.00$ & \\
\hline Community Leader Goals & $3.04(1.18)$ & $1.00-5.00$ & & $3.23(1.40)$ & $1.00-5.00$ & & $3.17(1.10)$ & $1.00-5.00$ & \\
\hline Fine Arts Goals & $2.94(1.61)$ & $1.00-5.00$ & & $2.62(1.45)$ & $1.00-5.00$ & & $3.08(1.49)$ & $1.00-5.00$ & \\
\hline Goals to have an easy life & $3.06(1.09)$ & $1.00-5.00$ & & $3.10(1.08)$ & $1.00-5.00$ & & $3.08(1.07)$ & $1.00-5.00$ & \\
\hline Goals to have fun & $4.70(.57)$ & $3.00-5.00$ & & $4.67(.63)$ & $2.00-5.00$ & & $4.52(.80)$ & $1.00-5.00$ & \\
\hline Higher Education Goals & $4.02(1.22)$ & $1.00-5.00$ & & $4.19(1.10)$ & $1.00-5.00$ & & $4.17(1.15)$ & $1.00-5.00$ & \\
\hline Parenthood Goals & $3.46(1.48)$ & $1.00-5.00$ & & $3.23(1.49)$ & 1.00 & & $3.23(1.52)$ & $1.00-5.00$ & \\
\hline Physical Condition Goals & $4.50(.72)$ & $2.00-5.00$ & & $4.52(.58)$ & $3.00-5.00$ & & $4.56(.54)$ & $3.00-5.00$ & \\
\hline Purpose Goals & $4.59(.84)$ & $1.00-5.00$ & & $4.58(.85)$ & $1.00-5.00$ & & $4.67(.88)$ & $1.00-5.00$ & \\
\hline Relationship Goals & $4.54(.97)$ & $1.00-5.00$ & & $4.67(.60)$ & $3.00-5.00$ & & $4.65(.60)$ & $3.00-5.00$ & \\
\hline Spiritual Life Goals & $2.72(1.57)$ & $1.00-5.00$ & & $2.85(1.58)$ & $1.00-5.00$ & & $2.81(1.63)$ & $1.00-5.00$ & \\
\hline Volunteer Goals & $3.89(1.08)$ & $1.00-5.00$ & & $3.73(1.16)$ & $1.00-5.00$ & & $3.75(1.06)$ & $1.00-5.00$ & \\
\hline Loneliness WUSTL & $1.59(.72)$ & $.67-3.50$ & .86 & $1.56(.69)$ & $.67-3.33$ & .81 & $1.60(.76)$ & $.67-3.33$ & .86 \\
\hline Purpose in Life & $4.65(.99)$ & $2.29-6.00$ & .84 & $4.47(.99)$ & $1.86-6.00$ & .83 & $4.38(.92)$ & $2.00-5.86$ & .80 \\
\hline Satisfaction with & 4.19 & $1.00-7.00$ & & $4.70(1.60)$ & $1.00-7.00$ & & $4.96(1.69)$ & $1.00-7.00$ & \\
\hline
\end{tabular}
Academics 
Table 3

Descriptive Statistics of Trait-Level Characteristics Across Waves

\begin{tabular}{|c|c|c|c|c|c|c|c|c|c|}
\hline \multirow{2}{*}{ Trait } & \multicolumn{3}{|c|}{ Wave 1} & \multicolumn{3}{|c|}{ Wave 2} & \multicolumn{3}{|c|}{ Wave 3} \\
\hline & M (SD) & Range & $\alpha$ & M (SD) & Range & $\alpha$ & M (SD) & Range & $\alpha$ \\
\hline $\begin{array}{c}\text { Satisfaction with } \\
\text { Community }\end{array}$ & $4.85(1.43)$ & $2.00-7.00$ & & $4.89(1.43)$ & $1.00-7.00$ & & $4.74(1.77)$ & $1.00-7.00$ & \\
\hline Satisfaction with Family & $5.67(1.77)$ & $1.00-7.00$ & & $5.66(1.67)$ & $1.00-7.00$ & & $5.70(1.53)$ & $1.00-7.00$ & \\
\hline Satisfaction with Finances & $3.87(1.89)$ & $1.00-7.00$ & & $4.15(1.96)$ & $1.00-7.00$ & & $4.38(1.91)$ & $1.00-7.00$ & \\
\hline $\begin{array}{c}\text { Satisfaction with } \\
\text { Friendships }\end{array}$ & $5.74(1.11)$ & $2.00-7.00$ & & $5.68(1.37)$ & $2.00-7.00$ & & $5.89(1.45)$ & $1.00-7.00$ & \\
\hline Satisfaction with Hobbies & $5.07(1.50)$ & $2.00-7.00$ & & $5.26(1.57)$ & $1.00-7.00$ & & $5.21(1.72)$ & $1.00-7.00$ & \\
\hline $\begin{array}{r}\text { Satisfaction with Physical } \\
\text { Health }\end{array}$ & $4.70(1.66)$ & $1.00-7.00$ & & $4.85(1.76)$ & $1.00-7.00$ & & $4.83(1.75)$ & $1.00-7.00$ & \\
\hline $\begin{array}{l}\text { Satisfaction with } \\
\text { Psychological Health }\end{array}$ & $4.22(2.04)$ & $1.00-7.00$ & & $4.66(1.72)$ & $1.00-7.00$ & & 4.57 (1.99) & $1.00-7.00$ & \\
\hline $\begin{array}{r}\text { Satisfaction with Romantic } \\
\text { Relationships }\end{array}$ & $4.24(2.06)$ & $1.00-7.00$ & & $4.13(2.09)$ & $1.00-7.00$ & & $4.09(2.06)$ & $1.00-7.00$ & \\
\hline Self-Control & $5.90(.83)$ & $3.86-8.00$ & .49 & $5.76(1.05)$ & $2.29-7.29$ & .67 & $5.55(.90)$ & $3.57-7.14$ & .58 \\
\hline Satisfaction with Life & $4.60(1.43)$ & $1.80-6.80$ & .85 & $4.83(1.38)$ & $1.60-7.00$ & .84 & $4.90(1.49)$ & $1.40-7.00$ & .90 \\
\hline
\end{tabular}

In addition, we asked participants to answer two questions about their experiences and changes in their experiences following the onset of COVID-19. Specifically, they were asked "What do you do now that did not do before COVID-19?" and "Which of your current behaviors are most different from your behaviors pre-COVID-19?" We also gave them space to add anything else they may want. Participants responses ranged in length, from 0 (chose not to respond; 5 participants) to 75 words. The median response length was 13 words. To better understand underlying themes of the text data, we removed filler (or stop) words (e.g., articles, conjunctions, etc.) and grouped words into meaningful phrases (e.g., "wear mask" or "don’t go out"). Next, we recoded similar phrases to match (e.g., "wearing mask" and "wear mask" became "wear mask") and examined how frequently such phrases were used across participants. A full table of the frequency of these recoded statements are in the online materials. The most frequent topic participants mentioned was "friends," which was mentioned by 19 participants. 10 
participants spoke about bout their "family" and being "home," which typically indicated their parents' home.

In coding these items, it became clear that there were overarching themes to many of the responses, so we next coded the matched words and phrases into broader categories, including hobbies, locations, social distancing, exercise, nonfamily members (particularly friends), media consumption, communication, family, sanitation practices, sleep, and productivity (see online materials for a full list).

Participants spoke at length about different hobbies they either had recently taken up or recently resumed. One participant wrote "I also decided to start learning Turkish because I am bored out of my mind (even though I am behind in literally all of my classes)." Others mentioned doing more reading for enjoyment, saying, for example, "I read more books, watch more TV, and engage in hobbies more." Others mentioned making exercise more focal, saying, for example, "I spend a lot more time working out because going for a run is my only way to get out of the house." Many spoke about combinations of hobbies, exercise, and time with family, such as, "I also play piano more often and take walks with my family more often." Such pursuits played into themes of productivity for some, such as one participant who wrote "Allow myself to be unproductive sometimes, play guitar, make specific creative plans for future."

Discussion of social distancing featured prominently in many participants writings, with one participant covering many common mentions: "wash my hands more often, wear a mask, wear gloves, go groceries shopping more often, prepare food myself." Or, more simply, many just reported staying home or rarely leaving their homes, saying, for example, "The main thing is just spending more time at home." 
Relatively few participants mentioned school or work. Among those that did, motivation and time spent on school appeared to be declining. One wrote, "Significantly less active and motivated, have extreme difficulty doing academic work vs. before I enjoyed it.” Another participant expressed anxiety about their future, writing "As a senior with no job lined up for after graduation, I have absolutely no idea if I'm still supposed to be looking for employment during this time. I originally wanted to get an internship for the summer, but it now seems to me that almost all internships are going to be cancelled or moved online. It therefore feels weird to apply and have interviews for internships/jobs that might not even happen...."

A number of participants expressed sadness, lethargy, and anxiety about their present and future experiences. One participant wrote, "I feel lethargic. Like life has come to a halt and I'm stuck in a ditch that I cant muster the energy to crawl out of," and another wrote, "I was generally a pretty happy, driven person before covid however as this has progressed, there have been days where I have been legitimately depressed and completely unmotivated.” Some reported mood swings, saying "My mood has changed a lot more frequently since being at home to COVID-19-- more frequent mood swings" or that they felt "More isolated, less extroverted, more temperamental."

Overall, in late April, participants expressed a range of situational, behavioral, social, and emotional responses to the COVID-19 related experiences and events. Given that participants described such changes in their lives, we next set to quantitatively estimating participants' personality coherence as measured in their everyday life before and after COVID-19 lockdowns began and assessed its consistency over time.

\section{Estimating Personality Coherence}


Next, we ran the GIMME procedure separately at each wave, which included unique uSEM models for each participant. The contemporaneous (within-time, lag 0) and lagged (across-time lag 1) estimates among personality states capture consistent patterns of each participant's experiences, which we treat as a proxy of coherence. Because previous research indicates relatively low reliability of lagged estimates (Beck \& Jackson, 2020b), we largely focus on contemporaneous results with full results for both lagged and contemporaneous results available in the online materials. Below, we discuss the model results of two sample participants to demonstrate their unique patterns of personality coherence (Figure 2). Notably, these two participants were those whose GIMME models were among the most consistent (Participant 33) and inconsistent (Participant 07) contemporaneous results. In the figure, each participant's layout in wave 2 has been constrained to match wave 1 to ease comparisons of their consistency of their coherence. The remaining figures are in the online materials, along with raw and formatted matrices of the results. 
Wave 1: Contemporaneous for S33

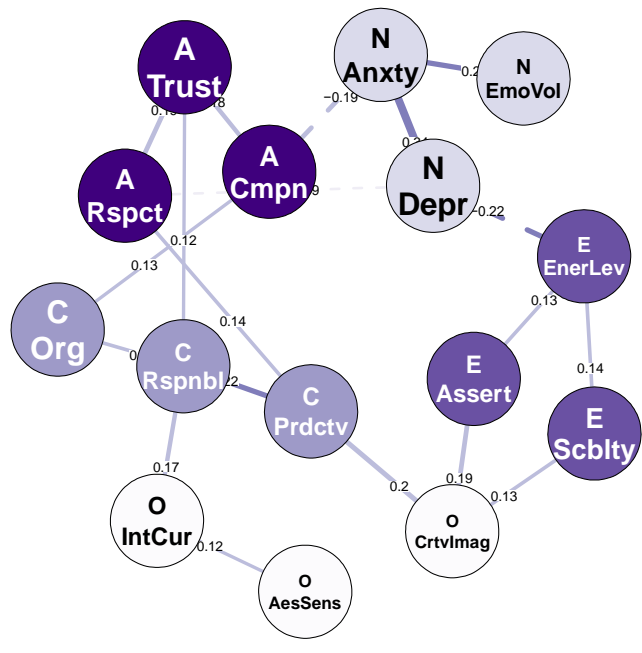

Wave 1: Contemporaneous for S07

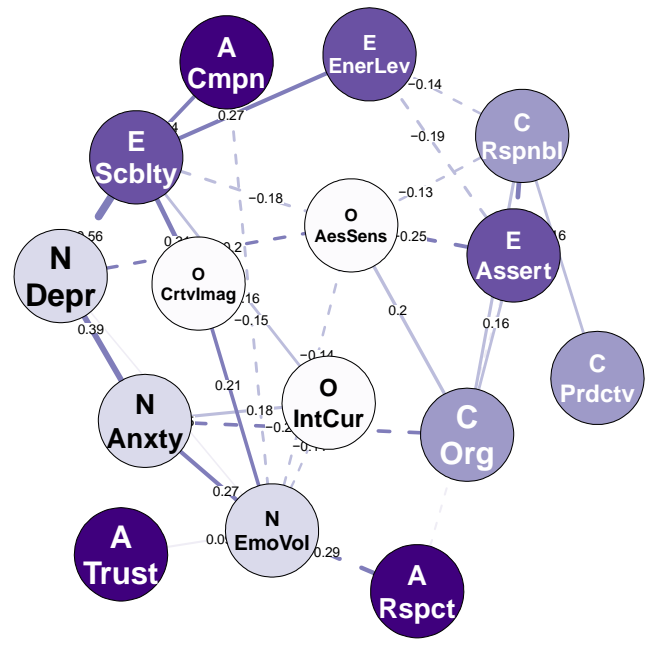

Wave 2: Contemporaneous for S33

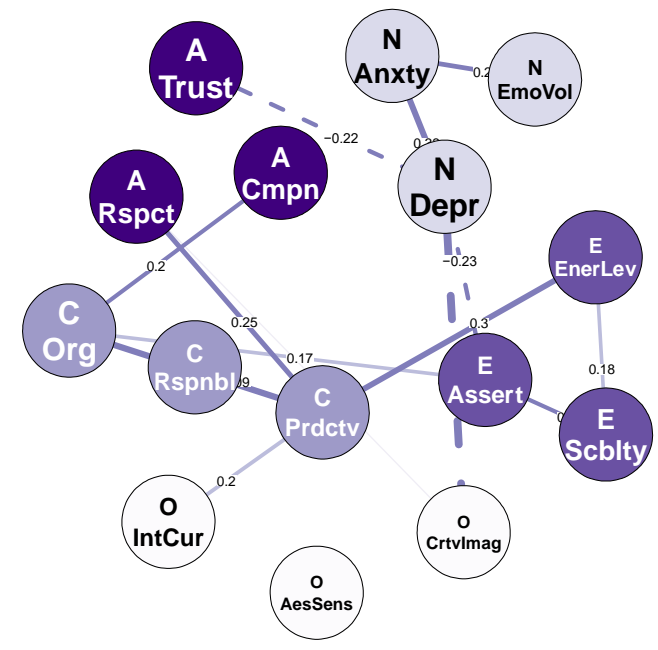

Wave 2: Contemporaneous for S07

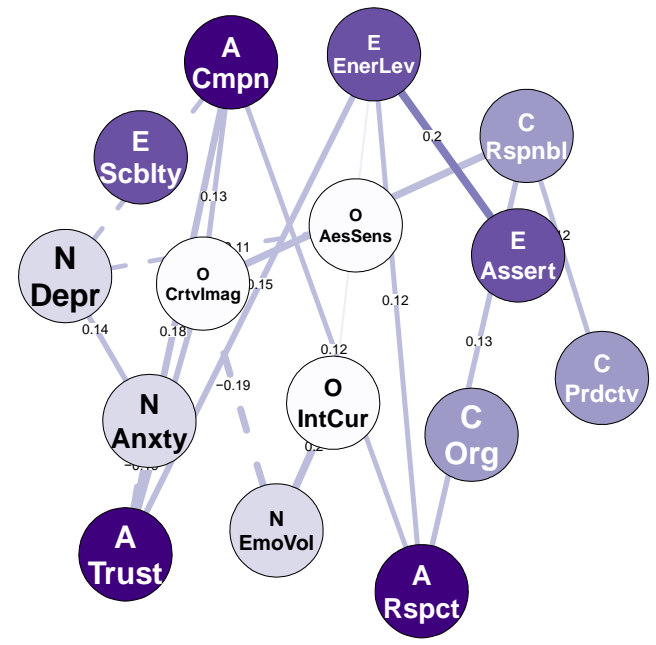

Figure 2. Sample contemporaneous model estimates represented as networks for two participants who had high ipsative consistency (top) or small (near 0) ipsative consistency (bottom). The left side of the figure contains wave 1 results and the right side contains wave 2 results. The color of the nodes represent to which Big Five factor an indicator putatively belongs and which Big Five factor it belongs to is represented by the capital letter $(\mathrm{E}, \mathrm{A}, \mathrm{C}, \mathrm{N}$, and $\mathrm{O})$ at the top of each circle. Dashed lines capture negative relationship, while solid lines capture positive relationship. Thicker lines are stronger (either positive or negative) relationships. Items are abbreviated due to space issues. Scblty $=$ Sociability, EnerLev $=$ Energy Levels, Assert $=$ Assertiveness, Cmpn = Compassion, Trust $=$ Trust, Rspct $=$ Respectfulness, Org $=$ Organization, Prdctv $=$ Productivity, Rspnbl $=$ Responsibility, Depr $=$ Depression, Anxty $=$ Anxiety, EmoVol $=$ Emotional Volatility, IntCur = Intellectual Curiosity, CrtvImag $=$ Creative Imagination, AesSens $=$ Aesthetic Sensitivity.

\section{Participant 33}


Participant 33's wave 1 network is in the upper left corner of Figure 2. Each of the circles represents one of the personality variables that participants responded to during the ESM protocol. The lines connecting each circle represents the partial relationship between two of the variables measured at the same time point - that is two experiences that were reliably experienced similarly over time. Solid lines indicate that the two tended to be experienced at similar levels while dashed lines indicate they tended to be experienced at opposite levels. The thickness of the lines indicates the strength of that association.

As is clear in Figure 2, items tended to cluster near other items that putatively belonged to the same trait. Participant 33's strongest associations were among indicators of Neuroticism, including between Anxiety $(\mathrm{N})$ and both Depression $(\mathrm{N} ; b=.34)$ and Emotional Volatility $(\mathrm{N} ; b$ $=.22$ ), as well as Conscientiousness, including between Responsibility and both Productivity (C; $b=.22)$ and Organization $(\mathrm{C} ; b=.14)$. Responsibility, in particular, was associated with a number of indicators of other traits, including Intellectual Curiosity $(\mathrm{O} ; b=.17)$ and Trust $(\mathrm{A} ; b$ $=12$ ). Although most associations were positive, there were a few notable negative associations, including between Depression $(\mathrm{N})$ and Energy Levels $(\mathrm{E} ; b=-.22)$ and between Anxiety $(\mathrm{N})$ and Compassion $(\mathrm{A} ; b=-.19)$.

Across waves, Participant 33 had a number of consistent associations. Associations among both Conscientiousness and Neuroticism persisted, with associations between Responsibility and Productivity $(\mathrm{C} ; b=.09)$ persisting and between Anxiety and Emotional Volatility $(\mathrm{N} ; b=.24)$ and Depression $(\mathrm{N} ; b=.33)$. However, there were a few notable changes. Rather than Creative Imagination (O) being positively associated with Productivity (C), Sociability (E), and Assertiveness (E) as in Wave 1, in Wave 2, it was associated only with feelings of Depression ( $\mathrm{N} ; b=-.44)$, such that more Creative Imagination was associated with 
less Depression, and very weakly with Respect $(\mathrm{A} ; b=.02)$. In addition, Intellectual curiosity at Wave 2 was positively associated with Productivity $(C ; b=.20)$, rather than with Responsibility as in Wave 1.

Participant 33's cross-wave consistency in coherent patterns of their personality was reflected in the free response behaviors and experiences they had in the early days after students were sent home and courses were moved online. Overall, Participant 33 was relatively consistent, and many of the changes observed in their empirical GIMME model cohere with the changes in their life they reported via free response. They reported keeping a similar bedtime but sleeping longer as well as keeping in touch with friends but via technology, which could drive some of the consistent associations among Conscientiousness indicators as well as the increased importance of Productivity at Wave 2. Some of their behavioral changes, however, including spending more time engaging in hobbies and reading as well as spending time with their family, could be reflected in changes between waves, such as negative associations between both Trust and Creative Imagination and reduced Depression.

\section{Participant 07}

Participant 07's Contemporaneous results are in the bottom row of Figure 2. As is clear in the Figure, relative to Participant 33, Participant 07's Big Five indicators did not clearly cluster with other indicators putatively belonging to the same between-person trait. This is reflected in that many of the strongest observed associations were across traits, with Sociability (E) predicting both lower Depression $(\mathrm{N} ; b=-.56)$ and higher Creative Imagination $(\mathrm{O} ; b=.31)$, and Assertiveness (E; $b=-.32)$ negatively predicting Responsibility (C). Emotional Volatility (N; 8), Sociability $(E ; 6)$ and Aesthetic Sensitivity $(O ; 6)$, in particular, had a large number of associations with other indicators, including each other. Lower Emotional Volatility was 
associated with higher Respectfulness (A; $b=-.29)$, Compassion (A; $b=-.15)$, Aesthetic Sensitivity (O), and Intellectual Curiosity $(\mathrm{O} ; b=-.11)$.

Unlike Participant 33, Participant 07 showed almost no cross-wave consistency. By closely examining the Figure, it becomes clear this is due to associations that were uniquely present in either wave and associations whose directions flipped. Indeed, Indicators, like Sociability, Aesthetic Sensitivity, and Emotional Volatility, that were associated with many other experiences in Wave 1 were associated with fewer indicators in Wave 2. While Sociability was associated with six other experiences in Wave 1, it was unassociated with any experiences in Wave 2. In addition, the negative association between Emotional Volatility $(\mathrm{N})$ and Intellectual Curiosity $(\mathrm{O})$ became strongly positive in Wave $2(b=.20)$. Moreover, relative to Wave 1 , Trust (A) became much more tied to other experiences. Agreeableness indicators, which had few associations with other indicators in Wave 1 had more associations with other indicators in Wave 2. For example, in Wave 1, Trust (A) was only weakly positively associated with Emotional Volatility (N; $b=.09)$, while in Wave 2, Trust (A) was positively associated with Energy Levels $(\mathrm{E} ; b=.15)$, Creative Imagination $(\mathrm{O} ; b=.14)$, and Compassion $(\mathrm{A} ; b=.18)$, and negatively associated with Anxiety ( $\mathrm{N} ; b=-.19)$. Compassion was similarly associated with lower Depression $(\mathrm{N} ; b=-.12)$ and more Creative Imagination $(\mathrm{O} ; b=.13)$.

In their text responses, Participant 07 reported that the biggest changes to their life following COVID-related lockdown was the loss of interaction with their friends. Before lockdown, they reported previously spending much time in person with their friends but made no mention of spending time with friends virtually. This change in time use and the importance of friendships may be reflected in how Sociability was an important indicator in Wave 1 but seemingly inconsequential in Wave 2. The lack of consistency may also be reflected in the way 
Participant 07 spoke about COVID-related changes. Unlike Participant 33, who spoke about the things they do now, many of which were different versions of previous behaviors, Participant 07 focused on the things they no longer do. As a result, Participant 07 may have experienced much larger changes in their everyday experiences.

\section{Longitudinal Personality Consistency}

After estimating coherence using the GIMME procedure, we calculated ipsative consistency of model coefficients for each individual across the two waves, which resulted in two estimates of longitudinal consistency as profile correlations (contemporaneous and lagged). These consistency estimates of profile correlations serve as our measure of personality coherence. Overall, as seen in Figure 3, estimates of consistency were small but reliably nonzero for contemporaneous $\left(M_{r}=.18 ; 95 \%\right.$ CI $\left.[.11, .22]\right)$ but less so for lagged $\left(M_{r}=.02 ; 95 \% \mathrm{CI}\right.$ $[-.01, .06])$ associations. Importantly, however, there were individual differences in consistency for both contemporaneous $\left(S D_{r}=.12 ; \min _{r}=-.12 ; \max _{r}=.37\right)$ and lagged consistency $\left(S D_{r}=\right.$ $\left..09 ; \min _{r}=-.21 ; \max _{r}=.18\right)$.

Because our main question was whether longitudinal consistency estimates of personality coherence matched a paradox of consistency (similar or greater consistency in the wake of great situational change) or accounts of change due to changes in environments (weaker consistency), a comparison sample is useful. Beck \& Jackson (2020b) reported two-year consistency estimates of a variety of methods for modeling personality coherence (i.e. idiographic structure) using time series data, including the GIMME procedure. In that study, 9 Big Five personality indicators were included, and, for analyses using GIMME models, they reported contemporaneous consistency $\left(M_{r}=.41 ; S D_{r}=.31 ; \min _{r}=-.31 ; \max _{r}=.89\right)$ and lagged consistency $\left(M_{r}=.07 ; S D_{r}\right.$ $\left.=.20 ; \min _{r}=-.30 ; \max _{r}=.55\right)$. To compare our results to theirs, we thus repeated our analyses 
with 9 analogous items. When doing so, we found that contemporaneous $\left(M_{r}=.31 ; S D_{r}=.24\right.$; $\left.\min _{r}=-.13 ; \max _{r}=.91\right)$ and lagged $\left(M_{r}=.08 ; S D_{r}=.22 ; \min _{r}=-.57 ; \max _{r}=.53\right)$ consistency were higher and much closer to GIMME consistency reported in Beck \& Jackson (2020b).

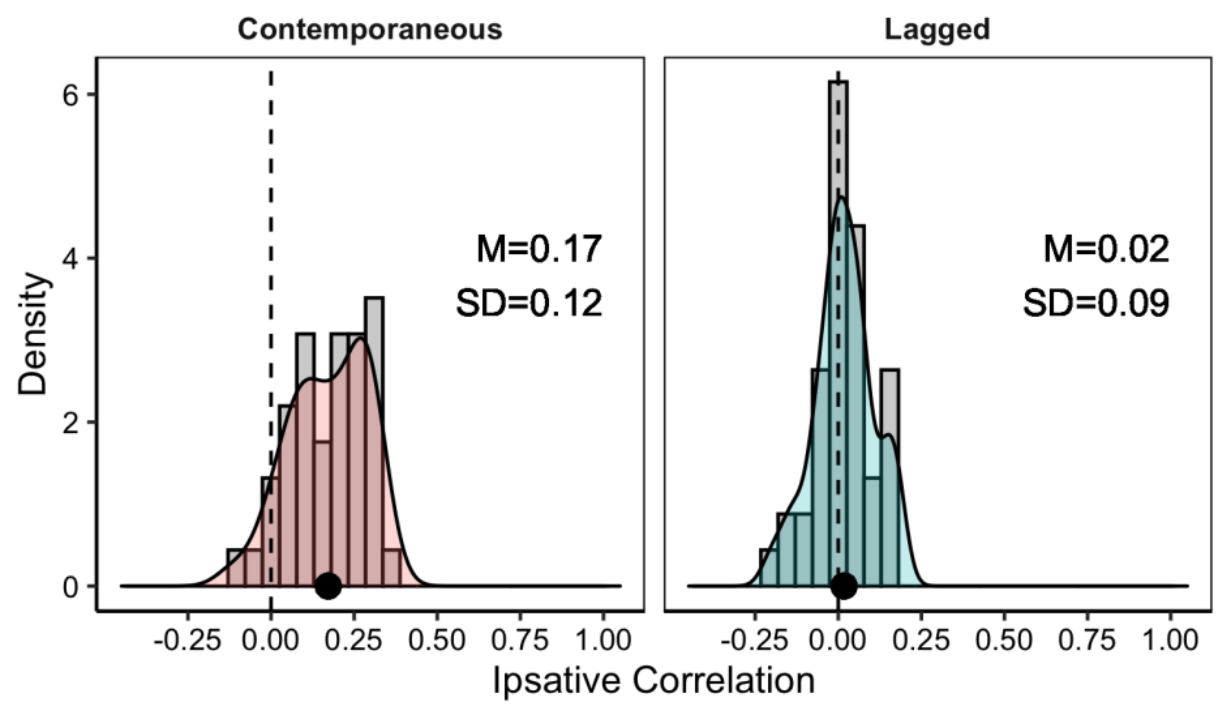

Figure 3. Density distributions of idiographic ipsative consistency in the wake of COVID-19. The left panel demonstrates contemporaneous (within-time) consistency, while the right panel demonstrates lagged (across-time) consistency.

\section{Antecedents of Personality Coherence}

After establishing that there were individual differences in consistency, we next examined whether participants' baseline characteristics predicted consistency. To do so, we built a series of models, predicting consistency from (1) the interval between ESM waves, (2) interval and wave 1 baseline characteristics, (3) interval, wave 1 characteristics, and wave 2 characteristics, and (4) interval, wave 1 and 2 characteristics, and the change in those characteristics across waves. As a reminder, Wave 1 occurred October 2018 and December 2019 immediately preceding each participant's first ESM period, and Wave 2 occurred between March 20-22, 2020. To ease interpretation of the coefficients, consistency was converted to $z$-scores using Fisher's $r$ to $z$ transformation and then scaled such that 0 indicated average consistency. 
Thus, a coefficient of .5 indicates that a one unit increase in the predictor is associated with a .5 standard deviation increase in consistency. The target parameter estimates for each of the key terms in each model are displayed in Table 4.

Table 4

Parameter Estimates of Target Terms for Models Estimating Ipsative Contemporaneous Consistency from Antecedents for Raw Data with 15 items

\section{Contemporaneous}

\begin{tabular}{|c|c|c|c|c|}
\hline Antecedent & $\begin{array}{c}\text { Interval } \\
\text { b [CI] }\end{array}$ & $\begin{array}{c}\text { W1 } \\
\text { b [CI] }\end{array}$ & $\begin{array}{c}\text { W2 } \\
\text { b [CI] }\end{array}$ & $\begin{array}{c}\text { W1 x W2 } \\
\text { b [CI] }\end{array}$ \\
\hline \multicolumn{5}{|l|}{ Affect } \\
\hline Negative Affect & $-.01[-.03,-.00]$ & $.08[-.06, .23]$ & $.16[-.05, .37]$ & $.04[-.08, .16]$ \\
\hline \multicolumn{5}{|l|}{ COVID Changes } \\
\hline COVID Worries & $-.01[-.03,-.00]$ & $.00[-.20, .20]$ & $.08[-.13, .29]$ & $.02[-.19, .24]$ \\
\hline Family In Person & $-.01[-.03,-.00]$ & $-.02[-.09, .06]$ & $.01[-.06, .08]$ & $.01[-.04, .07]$ \\
\hline Family On Phone & $-.02[-.03,-.00]$ & $-.09[-.21, .03]$ & $-.06[-.15, .03]$ & $-.00[-.06, .05]$ \\
\hline Family On Video & $-.01[-.03,-.00]$ & $-.08[-.18, .02]$ & $.06[-.04, .15]$ & $.00[-.05, .05]$ \\
\hline Friend In Person & $-.01[-.03,-.00]$ & $-.12[-.29, .06]$ & $-.01[-.09, .06]$ & $.07[-.04, .19]$ \\
\hline Friend On Phone & $-.01[-.03,-.00]$ & $-.03[-.12, .06]$ & $.01[-.11, .14]$ & $.00[-.06, .07]$ \\
\hline Friend On Video & $-.01[-.03,-.00]$ & $-.04[-.13, .06]$ & $.03[-.11, .16]$ & $.01[-.05, .07]$ \\
\hline \multicolumn{5}{|l|}{ Depression } \\
\hline \multicolumn{5}{|c|}{ 1 } \\
\hline Diligence & $-.01[-.03,-.00]$ & $-.25[-.46,-.03]$ & $.04[-.26, .33]$ & $.06[-.21, .33]$ \\
\hline Career & $-.01[-.03,-.00]$ & $-.22[-.43,-.01]$ & $.07[-.20, .36]$ & $.06[-.17, .28]$ \\
\hline Community Leader & $-.01[-.03,-.00]$ & $-.02[-.17, .13]$ & $-.10[-.24, .04]$ & $.03[-.08, .14]$ \\
\hline Fine Arts & $-.01[-.03,-.00]$ & $.02[-.09, .12]$ & $.06[-.10, .22]$ & $.08[-.01, .17]$ \\
\hline Have An Easy Life & $-.01[-.03,-.00]$ & $-.16[-.32,-.01]$ & $.00[-.19, .20]$ & $-.11[-.24, .01]$ \\
\hline Have Fun & $-.01[-.03,-.00]$ & $.04[-.23, .32]$ & $-.08[-.39, .24]$ & $-.22[-.70, .24]$ \\
\hline Higher Education & $-.01[-.03,-.00]$ & $-.09[-.22, .05]$ & $-.14[-.37, .09]$ & $-.04[-.19, .10]$ \\
\hline Parenthood & $-.01[-.03,-.00]$ & $-.06[-.17, .04]$ & $-.02[-.24, .19]$ & $-.03[-.13, .07]$ \\
\hline Physical Condition & $-.01[-.03,-.00]$ & $-.11[-.34, .13]$ & $-.21[-.56, .14]$ & $\begin{array}{c}-.49[-1.31 \\
.36]\end{array}$ \\
\hline Purpose & $-.01[-.03,-.00]$ & $-.09[-.28, .09]$ & $-.02[-.28, .25]$ & $-.04[-.17, .10]$ \\
\hline Relationship & $-.01[-.03,-.00]$ & $-.04[-.20, .12]$ & $-.03[-.31, .25]$ & $.10[-.09, .29]$ \\
\hline Spiritual Life & $-.01[-.03,-.00]$ & $.05[-.06, .16]$ & $-.05[-.20, .10]$ & $.06[-.03, .16]$ \\
\hline Volunteer & $-.01[-.03,-.00]$ & $-.03[-.18, .11]$ & $.10[-.10, .30]$ & $.00[-.11, .12]$ \\
\hline Loneliness & & & & \\
\hline
\end{tabular}




\section{Table 4}

Parameter Estimates of Target Terms for Models Estimating Ipsative Contemporaneous Consistency from Antecedents for Raw Data with 15 items

\section{Contemporaneous}

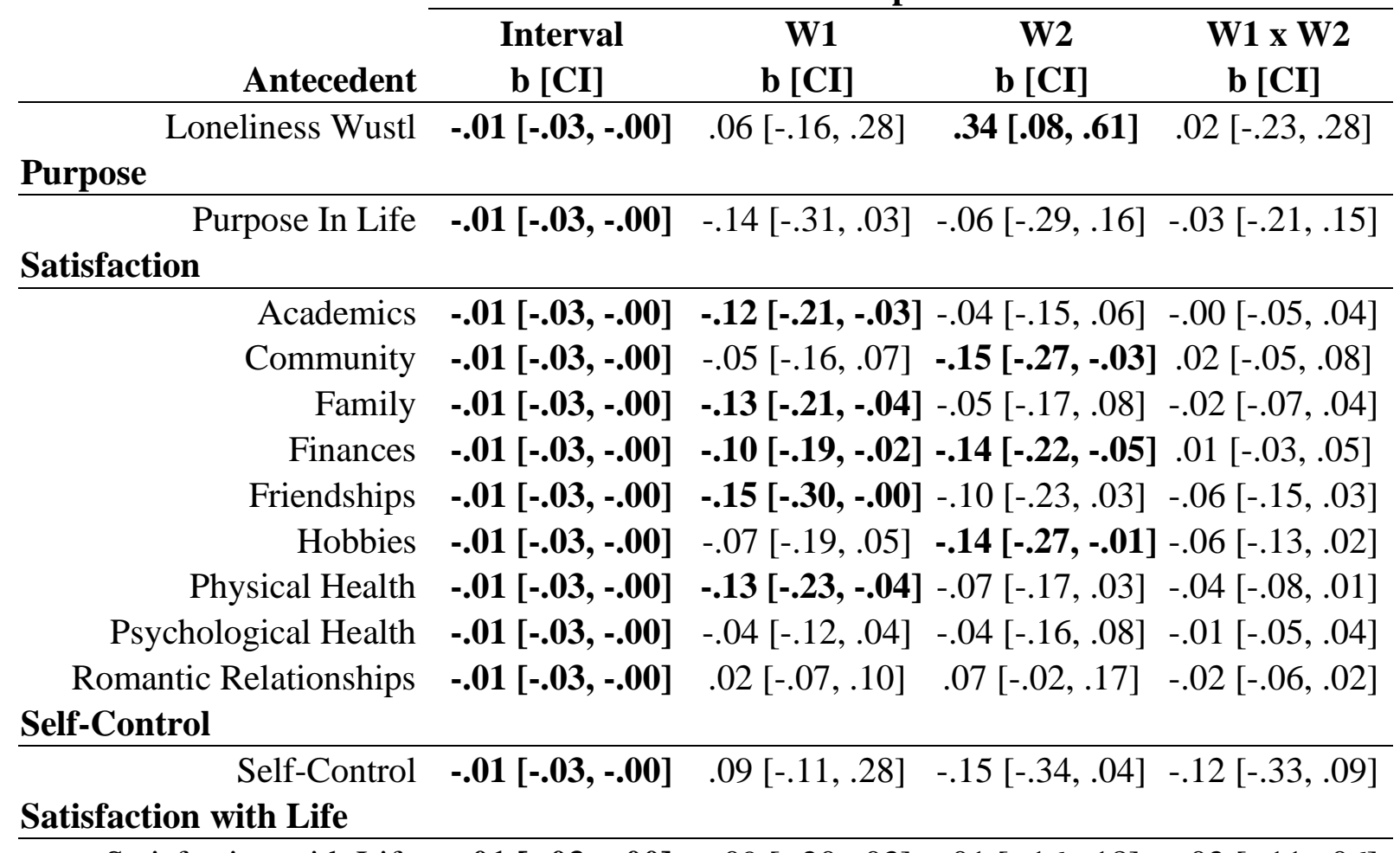

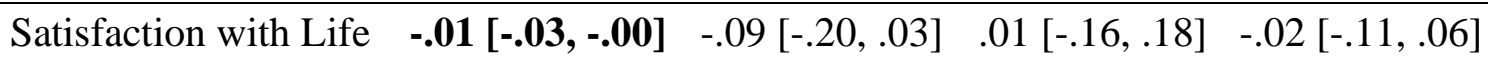

Note: Each column of estimates represents a separate model. Interval refers to the simple regressive association between the prediction interverbal and consistency. W1 refers to the association between wave 1 scores on each characteristic and consistency, controlling for prediction interval. W2 refers to the association between wave 2 scores on each characteristic and consistency, controlling for W1 scores and the prediction interval. W1 x W2 refers to the association between change in scores of each characteristic and consistency, controlling for W1 and W2 scores as well as the prediction interval.

Overall, there were few antecedents of consistency. Of the 280 target tests, 57 target terms were significant (20.4\%). Of these, 47 were for contemporaneous consistency, and the remaining nine were for lagged consistency (discussed in the online materials). Further, 35 of these 47 contemporaneous consistency antecedents were for the interval between measurement occasions, with longer intervals being associated with slightly smaller consistency estimates. The remaining only eight and four characteristics in Wave 1 and Wave 2, respectively predicted contemporaneous consistency. 
Results for measurement intervals can be seen in the far left column of Table 4. For each of these, the results demonstrate that longer intervals were associated with lower than average longitudinal consistency estimates. However, the effect size was quite small, indicating that each additional week between occasions was associated with about .01 standard deviations less consistency than average. The remaining antecedents of contemporaneous consistency largely reflected participants' satisfaction in specific domains. For example, higher levels of Wave 1 Satisfaction with Academics ( $b=-.12 ; 89 \%$ CI [-.03, -.00]), Family $(b=-.13 ; 89 \%$ CI [-.21,-.04 ]), Finances $(b=-.10 ; 89 \%$ CI [-.19, -.02]), Friendships $(b=-.15 ; 89 \%$ CI [-.30, -.00]), and Physical Health $(b=-.13 ; 89 \%$ CI $[-.23,-.04])$ as well as Diligence $(b=-.25 ; 89 \%$ CI [-.46, $.03])$ and Career Goals $(b=-.22 ; 89 \%$ CI $[-.43,-.01])$ and a Goal to Have an Easy Life $(b=-.16$; 89\% CI [-.32, -.01]) predicted lower contemporaneous consistency. In other words, individuals who scored one unit lower on each of these indicators tended to show between .10 and .30 standard deviations lower consistency.

In addition, higher levels of Wave 2 Satisfaction with Community $(b=-.15 ; 89 \%$ CI [$.27,-.03])$, Finances $(b=-.14 ; 89 \%$ CI [-.22, -.05]), and Hobbies $(b=-.14 ; 89 \%$ CI [-.27, -.01]) also predicted lower consistency above and beyond Wave 1 levels of each characteristic. Only Loneliness at the university predicted higher consistency, such that a one unit increase in loneliness was associated with a consistency estimate approximately .34 standard deviation higher $(b=.34 ; 89 \% \mathrm{CI}[.08, .61])$.

\section{Consequences of Personality Consistency}

Finally, we examined the consequences of consistency. To do so, we predicted later characteristics from consistency (converted to $z$-scores using Fisher's $r$ to $z$ transformation then z-scored in the sample), controlling for the interval between ESM waves. Thus, coefficients can 
be interpreted as the change in the outcome associated with a 1 SD change in consistency.

Overall, as seen in Table 5, there appeared to be relatively few consequences of coherence consistency. Overall, of the 76 tested associations, there were $9(11.8 \%)$ consistency estimates that predicted Wave 3 levels. Of these, two were estimates of contemporaneous consistency and seven were estimates of lagged consistency (see online materials for full lagged results). Above and beyond the interval between ESM measurement occasions, Contemporaneous consistency positively predicted higher Purpose Goals $(b=.38 ; 89 \%$ CI $[.10, .66])$ as well as more contact with family $(b=.70 ; 89 \%$ CI $[.16,1.26])$.

\section{Table 5}

Model Results of the Consequences of Contemporaneous Ipsative Idiographic Consistency in the Wake of COVID-19 for Raw Data with 15 items

\begin{tabular}{|c|c|c|c|}
\hline & & Con & poraneous \\
\hline & Measure & b & CI \\
\hline Affect & & & \\
\hline & Negative Affect & 0.25 & {$[-0.11,0.61]$} \\
\hline Depressio & & & \\
\hline & Depression & 0.10 & {$[-0.08,0.27]$} \\
\hline Diligence & & & \\
\hline & Diligence & 0.12 & {$[-0.08,0.32]$} \\
\hline Goals & & & \\
\hline & Career Goals & 0.24 & {$[-0.02,0.50]$} \\
\hline & Community Leader Goals & 0.29 & {$[-0.08,0.66]$} \\
\hline & Fine Arts Goals & -0.15 & {$[-0.61,0.32]$} \\
\hline & Goals to have an easy life & -0.00 & {$[-0.38,0.39]$} \\
\hline & Goals to have fun & 0.21 & {$[-0.08,0.51]$} \\
\hline & Higher Education Goals & 0.23 & {$[-0.13,0.57]$} \\
\hline & Parenthood Goals & -0.05 & {$[-0.49,0.37]$} \\
\hline & Physical Condition Goals & 0.19 & {$[-0.01,0.39]$} \\
\hline & Purpose Goals & 0.38 & {$[0.10,0.66]$} \\
\hline & Relationship Goals & 0.09 & {$[-0.14,0.33]$} \\
\hline & Spiritual Life Goals & 0.01 & {$[-0.46,0.46]$} \\
\hline & Volunteer Goals & 0.13 & {$[-0.24,0.50]$} \\
\hline Lonelines & & & \\
\hline & Loneliness WUSTL & 0.18 & {$[-0.03,0.39]$} \\
\hline Purpose & & & \\
\hline & Purpose in Life & 0.11 & {$[-0.13,0.35]$} \\
\hline
\end{tabular}


Table 5

Model Results of the Consequences of Contemporaneous Ipsative Idiographic Consistency in the Wake of COVID-19 for Raw Data with 15 items

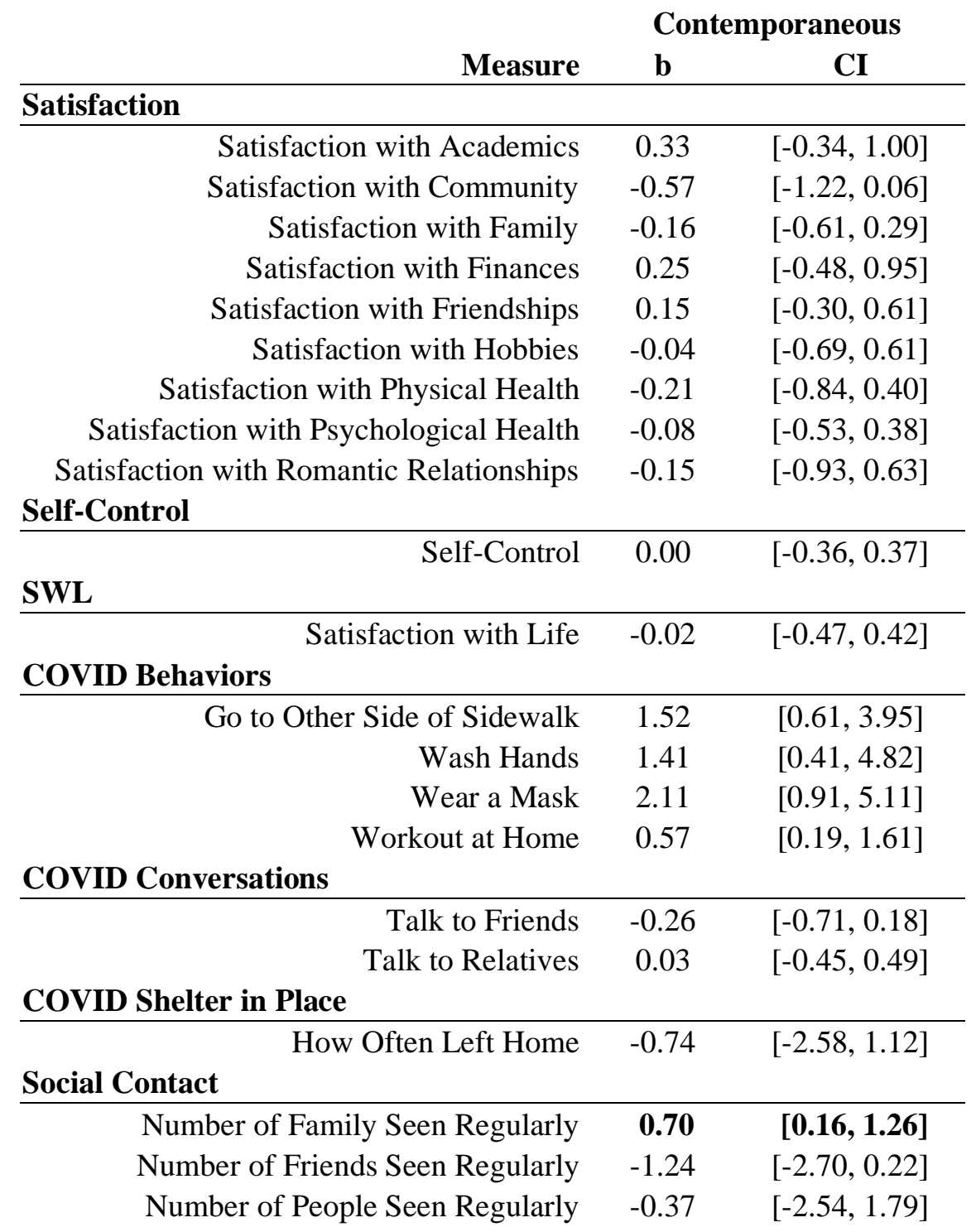

\section{Discussion}

The present study examined consistency and change of idiographic personality coherence following the onset of COVID-19. Using two waves of experience sampling method data - one before and one after the COVID-19 outbreak during stay at home orders - we examined the consistency and change in the idiographic personality coherence, as well as the antecedents and 
consequences of consistency. As such, we estimated personality coherence as a function of a major life upheaval that potentially could disrupt the personality system. Across waves, there was moderate consistency in contemporaneous associations and little consistency in lagged associations overall but strong individual differences in consistency. Despite the individual differences, there were few antecedents and consequences of consistency, and those that were observed tended to reflect individuals' goals and satisfaction. Overall, these findings suggest that personality coherence is influenced by the environment for some but not all people.

\section{Persons and Environments}

Almost no studies have examined personality coherence by modeling personality as sets of idiographic if...then contingencies or tested the consistency of them over time (c.f. Beck \& Jackson, 2020b; Fournier et al., 2008). Even those who have examined idiographic personality consistency and coherence have not done so in the midst of a global event. Thus, relative to previous investigations, the current study examines how a largescale global event, like the ongoing COVID-19 pandemic, may influence personality coherence and consistency. Traditional nomothetic trait and social cognitive perspectives posit that such environmental changes should lead to inconsistency in personality, but we expected personality to remain quite consistent in a so-called paradox of consistency (Caspi \& Moffitt, 1993). Despite this, the results were somewhat mixed. Consistency was relatively modest overall, and there were few antecedents and consequences of consistency. There are a few possible conclusions that can be drawn from these findings.

First, periods of great social change may not prompt individual to lean on established behavioral patterns (i.e. coherence) that elicit consistency in their personality over time. In other words, there may be no paradox of consistency. Instead, environmental shifts primarily serve to 
disrupt the personality system, leading to changes in personality coherence as both nomothetic and some social cognitive approaches suggest. When individuals have no normative guide or have degrees of uncertainty associated with them, they may innovate and test new patterns of behavior and demonstrate new forms of coherence. Especially in the early days of lockdown when individuals' previous lives were "on hold," many reported being unsure what to do with their time and adopted new hobbies like baking, scrapbooking, and exercising. Thus, these findings reiterate long standing views that major environmental shifts can lead to changes in daily thoughts, feelings, and behaviors.

Second, as with all psychological effects, there are likely individual differences. Given that there are so few studies of longitudinal idiographic personality consistency, the consistency seen in the present study may represent the upper bound of idiographic consistency. When we compared our results to the one previous study has looked at idiographic personality consistency using similar methods and a similar timeframe (see Beck \& Jackson, 2020b), we found relatively lower levels of consistency within our study when using the full 15-item set, but more similar levels when using a 9-item set matched to Beck and Jackson (2020b). In both sets, however, consistency remained modest in the aggregate. Despite this, not everyone demonstrated low levels of consistency, with some individuals showing considerable consistency and some showing almost none. Thus, it is possible that for some, the novel environment provided an opportunity for some to try out new behavioral patterns, which led to little to no consistency, while others persisted in similar patterns on the whole, which led to higher consistency. Some used the time as opportunity for growth, others bided their time until it was over, creating experiences that mimicked their previous life as much as possible. Consistent with this idea, a previous study that investigated how life events predict ipsative nomothetic personality 
consistency found that almost no major life events predicted individual differences in consistency (Jackson \& Beck, 2020). In other words, individual differences in coherence are not due to exogenous life experiences but are an individual difference in and of itself.

Third, previous conceptualizations of coherence may be thought of at a different level of analysis rather than the idiographic in situ, state assessments of personality in the current study. Coherence and consistency in personality as discussed by Caspi and Moffitt (1993) typically were conceptualized in terms of the absolute level or rank-order. Traditionally these are analyzed as between-person comparisons rather than idiographically. As a result, high levels of consistency could still emerge at the between-person level, even though we found relatively modest levels of average consistency when examined idiographically.

Fourth, if situations are thought to be critical for understanding patterns of behavior that largely underscore personality coherence, it may be necessary to include situations in models of idiographic personality directly (see Beck \& Jackson, 2020b). If patterns among personality indicators differ greatly depending on the context, then the relationship between these may differ depending on how often key situations were experienced during the ESM study period (see Jackson \& Beck, in press for an example). Thus, the models used in the current study may be the lower bound of coherence and consistency when not controlling for how situations influence if...then contingencies.

\section{Antecedents and Consequences of Coherence}

In the present study, we examined a number of pre-registered antecedents and consequences of coherence, including diligence, goals, satisfaction with life, and domain satisfaction. However, few of these were either antecedents or consequences of coherence. 
Understanding antecedents and consequences is critical because some previous research has indicated both that those with poorer psychological adjustment tend to show more nomothetic personality change (e.g., Klimstra, Noftle, Luyckx, Goossens, \& Robins, 2018) and that more nomothetic change and associated with poorer later psychological health (e.g., Human et al, 2013). In the present study, we found that some markers of adjustment, particularly satisfaction, were antecedents of consistency. However, each antecedent relationship between satisfaction and consistency was negative, with higher satisfaction with academics, family, finances, friendships, and physical health each predicting lower contemporaneous consistency. It could be that individuals who are more satisfied with their life and various domains of it may be more willing to take risks that ultimately change the structure of their if...then contingencies and, thus, their personality. In other words, individuals who were more satisfied may have been more likely to pursue personal growth in the wake of the COVID-19. On the lower end, individuals who are less satisfied may be so because they are stuck in maladaptive if...then's, making them unlikely to change those behaviors. Indeed, idiographic studies of personality and problems suggest that the two are difficult to disentangle (Jackson \& Beck, in press). Alternatively, it may be that those that are higher in satisfaction were more affected by the global pandemic, such that those who were more satisfied at early measurements felt more disruption as a result of lockdowns and restrictions.

Relative to antecedents, there were fewer consequences of consistency. Because consequences were not measured temporally distal to ESM measure, the present data may underestimate consequences of coherence. Given that consequences and outcomes tend to occur through accumulation, if consequences are not sufficiently distant, then any observed changes may represent the lower bound of changes. Thus, future research should examine whether 
idiographic ipsative coherence predicts more distal outcomes to a higher degree than less distal ones.

Despite this, our results partially supported the importance of consistency in predicting some goals that individuals pursue. We found that higher consistency predicted more pursual of goals related to one's physical condition and toward pursual of purpose in one's life. Thus, it may that in the wake of an environmental shift, individuals reached for their personal goals to guide behavior, making them more central in everyday behavior and leading to higher consistency. Alternatively, individuals who were more consistent may have found themselves more able to pursue goals to a greater degree than those who were less consistent. Taken with our previous proposal that more satisfied individuals may have less consistent because they pursued personal growth, this may indicate that these individuals did, indeed, pursue growth, at least in physical health and purpose domains.

In sum, there were relatively few antecedents and consequences of coherence, suggesting that individual differences in coherence may be due to other factors, including environmental shifts due to COVID-19. Despite this, goals and satisfaction were somewhat frequent antecedents or consequences, suggesting that they may be important in longitudinal behavioral coherence.

\section{Modeling Idiographic Coherence}

Idiographic methods and modeling have long been a core component of the study of personality coherence. Particularly when considered from a social cognitive perspective, idiographic approaches allow coherence to be considered with respect to an individual's unique patterns of behaviors and why they engage in them, which captures both descriptive and explanatory goals in personality. 
In the present study, we operationalized personality coherence as the associations among psychological phenomena experienced both together (i.e. at the same time, contemporaneous) and across time (i.e. one experience temporally follows another, lagged) using the GIMME procedure. Doing so allows us to understand both unique patterns of momentary experiences and well as how they unfold over time, similar to if...then contingency approaches in the social cognitive domain. However, rather than observing situation-behavior linkages (e.g., Wright \& Mischel, 1987) or asking individuals to report how they behave in specific situations (e.g., Bem \& Allen, 1974), the GIMME procedures allows these to be recovered analytically, thus coming closer to capturing probabilistic if...then relationships as has long been a goal of social cognitive approaches.

Despite the promise of the GIMME procedure for capturing contemporaneous (withintime) and lagged (across-time) associations, considerable challenges still remain in adequately and accurately uncovering idiographic personality coherence. First, a cornerstone of idiographic assessment is that participants are allowed to use idiographic constructs to describe their experiences. In other words, idiographic assessment does not require or assume that individuals will share in which phenomena capture their experiences. Investigations that have done so have revealed that individuals do reach for unique descriptors for themselves and that these show consistency over time (e.g., Jackson, Lord, Strube \& Harms, 2019).

Second, another important consideration in understanding personality, regardless of the level of aggregation (e.g., idiographic, within-person, between-person) is capturing how an individual's experiences unfold across time. To capture this, however, it is necessary to understand on what time course such experiences unfold. In the present study, for example, we pinged individuals every four hours, beginning at participant-provided wake times. Although 
some of the measured experiences, such as perhaps sociability, may unfold at such a timescale (i.e. we capture real peaks and valleys in individuals experiences of it), many psychological phenomena are thought to unfold on must faster time scales, including emotional experiences, like anxiety and depression.

Moreover, the problem of time scale is likely much more complicated than simply defining the time scale at which anxiety unfolds relative to sociability. Indeed, interindividual differences in such timescales have great implications for the frequency, latency, and duration of psychological experiences (see Revelle \& Condon, 2015). For example, even if two individuals are experiencing the same level of a phenomena at one time point, if they differ in the time course of it, then they may be at very different positions at a later time point (and have had a myriad of different levels and patterns of it in between the occasions). Despite this, at the time of this writing, there are no studies that explored the time scales at which psychological phenomena unfold, and the data used in the present study are inadequate to do so given their fixed interval data collection schedule. Thus, understanding how the timing of psychological phenomena unfolds remains a critical challenge for future quantitative research on personality coherence.

\section{Limitations and Future Directions}

The present study was the first to investigate personality coherence and consistency in the wake of a large-scale and global event, testing a popular theory about personality coherence heretofore untested systematically (Caspi \& Moffitt, 1993). Despite this, it was limited in a number of ways. First, we tested only one model for estimating idiographic personality structure, which may not be the optimal method for doing so for a number of reasons, including the issues of time scale discussed previously. To the extent that the model was non-optimal, this would likely decrease estimates of coherence. Future investigations should test idiographic coherence 
using other forms of models, such as change as outcome models (Danvers et al., 2020) and Bayesian Hierarchical Ornstein-Uhlenbeck Models (BHOUM; Oravecz, Tuerlinckx, \& Vandekerckhove, 2016), both of which are models meant to capture time-dependent associations among personality states from a dynamical systems perspective.

Second, although we excluded individuals with small numbers of observations, the remaining individuals still had a relatively small number of observations, on average, which could have led to unreliable estimates of model parameters and driven consistency estimates. Particularly given the procedures used to avoid lagging across non-contiguous time points (which should not be used to estimate lagged associations), the sample size for lagged estimates, in particular, may have been quite small. Future studies should endeavor to collect larger samples or utilize continuous time methods to avoid issues with lagged methods.

Third, the method used may not be optimal for capturing if...then's accurately. To the extent that it does not, it fails to capture personality coherence and will underestimate estimates of consistency. As noted in the previous section, the models estimated in the present study estimate contemporaneous (within-time) and lagged (across-time) estimates of how indicators relate to one another over time. There are three possible issues with such an approach. First, a lagged procedure is non-optimal when measures are taken at unequal intervals. Second, it estimates a static glimpse of general associations between manifestations of personality. Third, covariation among psychological states may not adequately capture the probabilist and complex relationships underlying if ...then contingencies. An alternative, dynamical systems perspective, ${ }^{5}$ however, examines the relationship between level and change on one or more indicators (see Danvers, Wundrack, \& Mehl, 2020, for a tutorial). In so doing, it can estimate the equilibria

\footnotetext{
${ }^{5}$ For a broader summary of additional dynamic methods for understanding time series data, see Wilt and Revelle (2021).
} 
points of a psychological system which are levels of certain experiences that a psychological system "prefers" to be at. Importantly, in contrast to static methods, this method can indicate multiple "preferred" levels, which would typically be captured inconsistently using the modeling procedure in the current study, which impact estimates of consistency.

Finally, we tested a subset of individual difference characteristics as antecedents and consequences of consistency and found few predictors of consistency. In general, there is a paucity of tests of antecedents and consequences of consistency outside of life events (e.g., Specht, Egloff, \& Schmukle, 2011). Thus, there is a critical need for further studies testing for antecedents and consequences of consistency.

\section{Conclusion}

The present study investigated coherence and consistency in idiographic personality in the wake of COVID-19 as well as antecedents and consequences. We found modest consistency in contemporaneous associations and weak consistency in lagged associations of personality across the onset of COVID-19, with relatively few antecedents and consequences of consistency. Most of the observed antecedents corresponded to satisfaction, which most of the consequences corresponded to goals, suggesting that satisfaction and goals may be critical for personality coherence and consistency by directing behavior. 


\section{Data Accessibility Statement}

This study was preregistered on the Open Science Framework (OSF; https://osf.io/qjs5v) and all data, analysis scripts, and results are available on both the OSF (https://osf.io/qwtu3/) and GitHub (https://github.com/emoriebeck/covid_consistency). 


\section{References}

Allport, G. W. (1921). Personality and character. Psychological Bulletin, 18(9), 441455. https://doi.org/10.1037/h0066265

Allport, G. W. (1937). Personality: a psychological interpretation. Holt.

Beck, E. D. \& Jackson, J. J. (2020a). Within-person variability. In Rauthmann, J., editor, Handbook of Personality Dynamics and Processes. Elsevier, 1st edition. https://doi.org/10.1016/B978-0-12-813995-0.00001-7

Beck, E. D., \& Jackson, J. J. (2020b). Consistency and change in idiographic personality: A longitudinal ESM network study. Journal of Personality and Social Psychology, 118(5), 108. https://doi.org/10.1037/pspp0000249

Beck, E. D., \& Jackson, J. J. (2020c). Idiographic Traits: A Return to Allportian Approaches to Personality. Current Directions in Psychological Science, 29(3), 301-308. https://doi.org/10.1177/0963721420915860

Bem, D. J., \& Funder, D. C. (1978). Predicting more of the people more of the time: Assessing the personality of situations. Psychological Review, 85(6), 485-501. https://doi.org/10.1037/0033-295X.85.6.485

Bernard, H., \& Schuttenberg, E. M. (1995). Development of the Diligence Inventory-higher education form. Journal of Research \& Development in Education.

Bleidorn, W., Hopwood, C. J., \& Lucas, R. E. (2018). Life events and personality trait change. Journal of Personality, 86(1), 83-96. https://doi.org/10.1111/jopy.12286

Bleidorn, W., Kandler, C., Riemann, R., Angleitner, A., \& Spinath, F. M. (2012). Genetic and environmental influences on personality profile stability: Unraveling the normativeness 
problem. Journal of Personality, 80(4), 1029-106. https://doi.org/10.1111/j.14676494.2011.00758.x

Briley, D. A., \& Tucker-Drob, E. M. (2017). Comparing the developmental genetics of cognition and personality over the life span. Journal of Personality, 85(1), 51-64. https://doi.org/10.1111/jopy.12186

Bürkner, P. C., \& Buerkner, M. P. C. (2016). Package 'brms'.

Caspi, A., \& Moffitt, T. E. (1993). When do individual differences matter? A paradoxical theory of personality coherence. Psychological Inquiry, 4(4), 247-271. https://doi.org/10.1207/s15327965pli0404_1

Cervone, D. (2005). Personality architecture: Within-person structures and processes. Annu. Rev. Psychol., 56, 423-452. https://doi.org/10.1146/annurev.psych.56.091103.070133

Damian, R. I., Spengler, M., Sutu, A., \& Roberts, B. W. (2019). Sixteen going on sixty-six: A longitudinal study of personality stability and change across 50 years. Journal of Personality and Social Psychology, 117(3), 674-695. https://doi.org/10.1037/pspp0000210

Danvers, A. F., Wundrack, R., \& Mehl, M. (2020). Equilibria in Personality States: A Conceptual Primer for Dynamics in Personality States. European Journal of Personality, 34 999- 1016. https://doi.org/10.1002/per.2239

De Leeuw, J. R. (2015). jsPsych: A JavaScript library for creating behavioral experiments in a Web browser. Behavior research methods, 47(1), 1-12. https://doi.org/10.3758/s13428014-0458-y 
Diener, E. D., Emmons, R. A., Larsen, R. J., \& Griffin, S. (1985). The satisfaction with life scale. Journal of Personality Assessment, 49(1), 71-75. https://doi.org/10.1207/s15327752jpa4901_13

Donnellan, M. B., Lucas, R. E., \& Fleeson, W. (2009). Introduction to personality and assessment at age 40: Reflections on the legacy of the person-situation debate and the future of person-situation integration [Editorial]. Journal of Research in Personality, 43(2), 117-119. https://doi.org/10.1016/j.jrp.2009.02.010

Emmons, R. A., \& Diener, E. (1985). Personality correlates of subjective well-being. Personality and Social Psychology Bulletin, 11(1), 89-97. https://doi.org/10.1177/0146167285111008

Emmons, R. A., \& Diener, E. (1986). Situation selection as a moderator of response consistency and stability. Journal of Personality and Social Psychology, 51(5), 1013-1019. https://doi.org/10.1037/0022-3514.51.5.1013

Epstein, S. (1979). The stability of behavior: I. On predicting most of the people much of the time. Journal of Personality and Social Psychology, 37(7), 1097-1126. https://doi.org/10.1037/0022-3514.37.7.1097

Fleeson, W., \& Noftle, E. (2008). The end of the person-situation debate: An emerging synthesis in the answer to the consistency question. Social and Personality Psychology Compass, 2(4), 1667-1684. https://doi.org/10.1111/j.1751-9004.2008.00122.x

Fournier, M. A., Moskowitz, D. S., \& Zuroff, D. C. (2008). Integrating dispositions, signatures, and the interpersonal domain. Journal of personality and social psychology, 94(3), 531545. https://doi.org/10.1037/0022-3514.94.3.531 
Funder, D. C. (2006). Towards a resolution of the personality triad: Persons, situations, and behaviors. Journal of Research in Personality, 40(1), 21-34. https://doi.org/10.1016/j.jrp.2005.08.003

Furr, R. M., \& Funder, D. C. (2018). Persons, situations, and person-situation interactions. Handbook of personality: Theory and research, 1-42.

Honaker, J., King, G., \& Blackwell, M. (2011). Amelia II: A program for missing data. Journal of Statistical Software, 45(7), 1-47.

Human, L. J., Biesanz, J. C., Miller, G. E., Chen, E., Lachman, M. E., \& Seeman, T. E. (2013). Is change bad? Personality change is associated with poorer psychological health and greater metabolic syndrome in midlife. Journal of Personality, 81(3), 249-260. https://doi.org/10.1111/jopy.12002

Jackson, J. J. \& Beck, E. D. (2020). Personality development beyond the mean: Do life events shape personality variability, structure, and ipsative continuity. The Journals of Gerontology, Series B: Psychological Sciences and Social Sciences. 76(1), 20-30. https://doi.org/10.1093/geronb/gbaa093

Jackson, J. J. \& Beck, E. D (in press). Using idiographic models to distinguish personality and psychopathology. Journal of Personality.

Jackson, J. J., Lord, A. N., Strube, M. J., \& Harms, P.D (2019). An Individual, idiographic approach to personality development: Examining change and stability in self-concept. https://psyarxiv.com/qdp98/

Jackson, J. J., Thoemmes, F., Jonkmann, K., Lüdtke, O., \& Trautwien, U. (2012). Military training and personality trait development: Does the military make the man or does the 
man make the military? Psychological Science, 23, 270 - 277.

https://doi.org/10.1177/0956797611423545

Klimstra, T. A., Noftle, E. E., Luyckx, K., Goossens, L., \& Robins, R. W. (2018). Personality development and adjustment in college: A multifaceted, cross-national view. Journal of Personality and Social Psychology, 115(2), 338-361.

https://doi.org/10.1037/pspp0000205

Lane, S., Gates, K. M., Molenaar, P., Hallquist, M., \& Pike, H. (2016). Gimme: Group iterative multiple model estimation. Computer Software. Retrieved from, https://CRAN. R-project. org/package $=$ gimme .

Lane, S. T., Gates, K. M., Pike, H. K., Beltz, A. M., \& Wright, A. G. (2019). Uncovering general, shared, and unique temporal patterns in ambulatory assessment data. Psychological Methods, , 24(1), 54-69. https://doi.org/10.1037/met0000192

Lodi-Smith, J., \& Roberts, B. W. (2007). Social investment and personality: A meta-analysis of the relationship of personality traits to investment in work, family, religion, and volunteerism. Personality and Social Psychology Review, 11(1), 68-86. https://doi.org/10.1177/1088868306294590

Luhmann, M., Orth, U., Specht, J., Kandler, C., \& Lucas, R. E. (2014). Studying changes in life circumstances and personality: It's about time. European Journal of Personality, 28(3), 256-266. https://doi.org/10.1002/per.1951

Mischel, W. (1973). Toward a cognitive social learning reconceptualization of personality. Psychological Review, 80(4), 252-283. https://doi.org/10.1037/h0035002 
Mischel, W., \& Peake, P. K. (1982). Beyond déjà vu in the search for cross-situational consistency. Psychological Review, 89(6), 730-755. https://doi.org/10.1037/0033295X.89.6.730

Mischel, W., \& Shoda, Y. (1995). A cognitive-affective system theory of personality: reconceptualizing situations, dispositions, dynamics, and invariance in personality structure. Psychological review, 102(2), 246-68. doi: 10.1037/0033-295x.102.2.246

Moffitt, T. E., \& Caspi, A. (2001). Childhood predictors differentiate life-course persistent and adolescence-limited antisocial pathways among males and females. Development and Psychopathology, 13(2), 355-375. https://doi.org/10.1017/S0954579401002097

Mõttus, R., Wood, D., Condon, D. M., Back, M. D., Baumert, A., Costantini, G., ... \& Murray, A. (2020). Descriptive, predictive and explanatory personality research: Different goals, different approaches, but a shared need to move beyond the Big Few traits. European Journal of Personality, 34(6), 1175-1201. https://doi.org/10.1002/per.2311

Murray, H. A. (1938). Explorations in personality: a clinical and experimental study of fifty men of college age. Oxford Univ. Press.

Neto, F. (1992). Loneliness among Portuguese adolescents. Social Behavior and Personality, 20, 15-22. https://doi.org/10.2224/sbp.1992.20.1.15

Neyer, F. J., Mund, M., Zimmermann, J., \& Wrzus, C. (2014). Personality-relationship transactions revisited. Journal of Personality, 82(6), 539-55. https://doi.org/10.1111/jopy.12063

Ogle, C. M., Rubin, D. C., \& Siegler, I. C. (2014). Changes in neuroticism following trauma exposure. Journal of Personality, 82(2), 93-102. https://doi.org/10.1111/jopy.12037 
Oravecz, Z., Tuerlinckx, F., \& Vandekerckhove, J. (2016). Bayesian data analysis with the bivariate hierarchical Ornstein-Uhlenbeck process model. Multivariate Behavioral Research, 51(1), 106-119. https://doi.org/10.1080/00273171.2015.1110512

Perugini, M., \& Conner, M. (2000). Predicting and understanding behavioral volitions: The interplay between goals and behaviors. European Journal of Social Psychology, 30(5), 705-731. https://doi.org/10.1002/1099-0992(200009/10)30:5<705::AIDEJSP18>3.0.CO;2-\%23

R Core Team (2019). R: A language and environment for statistical computing. R Foundation for Statistical Computing, Vienna, Austria. URL https://www.R-project.org/.

Radloff, L. S. (1977). The CES-D scale: A self report depression scale for research in the general population. Applied Psychological Measurements, 1, 385-401. https://doi.org/10.1177/014662167700100306

Revelle, W. R. (2017). psych: Procedures for personality and psychological research.

Revelle, W., \& Condon, D. M. (2015). A model for personality at three levels. Journal of Research in Personality, 56, 70-81. https://doi.org/10.1016/j.jrp.2014.12.006

Revelle, W., Condon, D. M., Wilt, J., French, J. A., Brown, A., \& Elleman, L. G. (2016). Web and phone based data collection using planned missing designs. Sage handbook of online research methods (2nd ed., p. 578-595). Sage Publications, Inc. https://dx.doi.org/10.4135/9781473957992.n33

Roberts, B. W., Donnellan, M. B., \& Hill, P. L. (2012). Personality trait development in adulthood. Handbook of Psychology, Second Edition, 5. https://doi.org/10.1007/978-0306-48247-2_26 
Roberts, B. W., \& Jackson, J. J. (2008). Sociogenomic personality psychology. Journal of Personality, 76(6), 1523-1544. https://doi.org/10.1111/j.1467-6494.2008.00530.x

Roberts, B. W., \& Robins, R. W. (2000). Broad dispositions, broad aspirations: The intersection of personality traits and major life goals. Personality and Social Psychology Bulletin, 26(10), 1284-1296. https://doi.org/10.1177/0146167200262009

Robins, R. W., Fraley, R. C., Roberts, B. W., \& Trzesniewski, K. H. (2001). A longitudinal study of personality change in young adulthood. Journal of Personality, 69(4), 617-640. https://doi.org/10.1111/1467-6494.694157

Ryff, C. D. (1989). Happiness is everything, or is it? Explorations on the meaning of psychological well-being. Journal of Personality and Social Psychology, 57(6), 1069. DOI: $10.1037 / 0022-3514.57 .6 .1069$

Soto, C. J., \& John, O. P. (2017). The next Big Five Inventory (BFI-2): Developing and assessing a hierarchical model with 15 facets to enhance bandwidth, fidelity, and predictive power. Journal of Personality and Social Psychology, 113(1), 117-143. https://doi.org/10.1037/pspp0000096

Specht, J., Egloff, B., \& Schmukle, S. C. (2011). Stability and change of personality across the life course: the impact of age and major life events on mean-level and rank-order stability of the Big Five. Journal of Personality and Social Psychology, 101(4), 862-882. https://doi.org/10.1037/a0024950

Revelle, W., \& Wilt, J. (2021). The history of dynamic approaches to personality. In The Handbook of Personality Dynamics and Processes (pp. 3-31). Academic Press. 
Wright, J. C., \& Mischel, W. (1987). A conditional approach to dispositional constructs: the local predictability of social behavior. Journal of Personality and Social Psychology, 53(6), 1159-1177. https://doi.org/10.1037/0022-3514.53.6.1159 